\title{
REDUCTION OF PRESYMPLECTIC MANIFOLDS WITH SYMMETRY
}

\author{
A. Echeverría-Enríquez, M. C. Muñoz-Lecanda* N. Román-RoY ${ }^{\dagger}$ \\ Departamento de Matemática Aplicada y Telemática \\ Campus Norte U.P.C., Módulo C-3 \\ C/ Jordi Girona 1 \\ E-08034 Barcelona, Spain
}

To be published in Rev. Math. Phys. 11(10) 1999

\begin{abstract}
Actions of Lie groups on presymplectic manifolds are analyzed, introducing the suitable comomentum and momentum maps. The subsequent theory of reduction of presymplectic dynamical systems with symmetry is studied. In this way, we give a method of reduction which enables us to remove gauge symmetries as well as non-gauge "rigid" symmetries at once. This method is compared with other step-by-step reduction procedures. As particular examples in this framework, we discuss the reduction of time-dependent dynamical systems with symmetry, the reduction of a mechanical model of field theories with gauge and non-gauge symmetries, and the gauge reduction of the system made of a conformal particle.
\end{abstract}

Key words: Presymplectic manifolds, Lie groups, Momentum maps, Symmetries, Reduction.

AMS s.c. (1991): 57S25, 58D19, 70H33. PACS: 0240, 0320

${ }^{*} \mathbf{e}-$ mail: MATMCML@MAT.UPC.ES

${ }^{\dagger} \mathbf{e}$-mail: MATNRR@MAT.UPC.ES 


\section{Introduction}

The problem of reduction of dynamical systems with symmetry has deserved the interest of theoretical physicists and mathematicians, with the purpose of reducing the number of evolution equations, by finding first integrals of motion. In particular, geometric treatment of this subject has been revealed as a powerful tool in the study of this question. The pioneering and fundamental work on this topic has been carried out by Marsden and Weinstein [42] (see also [1], 34] and [52]). They demonstrated that, for a free and proper symplectic action of a (connected) Lie group on a (connected) symplectic manifold (which is the phase space of an autonomous regular Hamiltonian system with symmetry), and a weakly regular value of the momentum map associated with this action, the reduced phase space has a structure of symplectic manifold and inherits a Hamiltonian dynamics from the initial system.

Nevertheless, the problem of reduction can appear under many different aspects. Subsequently, other authors have investigated aspects of the theory of reduction for other particular cases.

Thus, for instance, if zero is a singular value of the momentum map (in a symplectic manifold) then the Marsden-Weinstein technique gives a reduced phase space which is a stratified symplectic space [49]. Starting from this result, reduction of time-dependent regular Hamiltonian systems with momentum mappings with singular value at zero is achieved in [33], where, using the extended phase space symplectic formalism, it is proved that the reduced phase space is also a stratified space but with a cosymplectic structure. Another approach to the problem of singular values can be found in 沺 (see also other references quoted therein), where reduction of symplectic manifolds at singular values of the momentum mapping is considered, showing that, under certain conditions, the reduced space inherits a non-degenerate Poisson structure. However, research in this area is not yet complete.

In the realm of momentum maps with regular values, the Marsden-Weinstein symplectic reduction scheme has been applied to many different situations. For example, reduction of timedependent regular Hamiltonian systems is developed in the framework of cosymplectic manifolds in [3], obtaining a reduced phase space which inherits a structure of cosymplectic manifold. The study of autonomous singular Lagrangian systems can be found in [9] and, in particular, the conditions for the reduced phase space to inherit an almost-tangent structure are studied for certain kinds of degenerate Lagrangians. Some of the results here obtained are generalized to the case of nonautonomous singular Lagrangian systems and for a larger class of degenerate Lagrangians in 28]. Another approach to this question is made in [32], where the authors analyze the conditions for the existence of a regular Lagrangian function in the reduced phase space obtained after reduction, in such a way that the reduced cosymplectic or contact structure (and hence the reduced Hamiltonian function) can be constructed from it.

Furthermore, there are other situations in reduction theory. So, for instance, the theory of reduction of Poisson manifolds is treated in works such as [31] and 39]. Reduction of cotangent bundles of Lie groups within semidirect products is considered in 40, with several applications to outstanding problems in mathematical physics. Concerning the subject of Lagrangian reduction, there are some works, such as 41], which consider the problem from the point of view of reducing variational principles (instead of reducing the almost tangent structure, as it is made in some of the above mentioned references). Finally, the study of reduction of non-holonomic systems can be found, for instance, in [6], 10] and [36]. (Of course, this list of references is far to be complete).

The aim of this work is to apply the Marsden-Weinstein method to reduce presymplectic manifolds with Lie groups of symmetries acting on them. The interest of this topic lies in the fact that the geometrical description of many dynamical systems is given by means of presymplectic mani- 
folds. One of the more frequent cases is the Lagrangian formalism of singular mechanical systems, where the phase space is the manifold $\mathrm{T} Q$ ( $Q$ being the configuration manifold of the system), endowed with the presymplectic form $\Omega_{\mathcal{L}}$, which is constructed from the singular Lagrangian function $\mathcal{L}$. Other typical examples are certain descriptions of non-autonomous mechanical systems (both in the Lagrangian and Hamiltonian formalism), where the phase space is a contact (cosymplectic) manifold. Certainly, these kind of systems could be reduced by first constructing an ambient symplectic manifold where the system is coisotropically imbedded, and then applying the symplectic reduction procedure to it [29]. But we give a reduction procedure that allows us to implement the Marsden-Weinstein technique directly for the initial presymplectic system.

In particular, we construct comomentum and momentum mappings for presymplectic actions of Lie groups, analyzing the obstruction to their existence and studying some characteristics features of the level sets of the momentum map. Then, we prove that, for weakly regular values of this momentum map, and under the usual suitable assumptions, the reduced phase space inherits a presymplectic structure. Next we apply these results in order to reduce presymplectic dynamical systems with symmetry, showing that, if we consider together gauge and non-gauge ("rigid") symmetries, and we reduce the system by all of them, then this procedure leads to the same results as if we first remove the gauge redundancy and then reduce the remaining "rigid" symmetries. Finally, we analyze three examples, namely: non-autonomous dynamical systems with symmetry (comparing then the results so obtained with those of some of the above mentioned references), a mechanical model for field theories, and the conformal particle.

The paper is organized in the following way:

The first part is devoted to the study of presymplectic group actions. Thus, in sections 2.1 and 2.2, we review some basic concepts on presymplectic manifolds and present the actions of Lie groups on them. In sections 2.3 and 2.4 we define the comomentum and momentum mappings for this kind of actions, studying the obstruction to their existence, the level sets of the momentum map and their reduction.

The second part deals with symmetries of presymplectic dynamical systems. First, in section 3.1, we review the basic features of this kind of dynamical systems. Section 3.2 is devoted to defining and analyzing the concept of symmetry for these systems and to establish the reduction procedure for compatible presymplectic systems. The reduction procedure for non-compatible presymplectic systems and its characteristic features is established in section 3.5. This part ends with a comparative study between this reduction method and other different ways for reducing presymplectic systems, which is performed in sections 3.3 and 3.4 .

In the third part some examples are analyzed. In sections 4.1 and 4.2 these techniques are applied in order to make the reduction of non-autonomous systems with symmetry and, as a particular example, the dynamics of autonomous regular dynamical systems is obtained in this context. A further example is the complete reduction of a particular case of a mechanical model of field theories coupled to external fields (due to Capri and Kobayashi), which is investigated in sections 4.3 and 4.4 . As the last example, the gauge reduction of the system of a conformal particle is discussed, in this framework, in section 4.5 .

Finally, we discuss the results and compare them with those obtained in some of the works above mentioned.

An appendix is devoted to a linear interpretation of the reduction theory.

All the manifolds are real, connected, second countable and $\mathrm{C}^{\infty}$. The maps are assumed to be $\mathrm{C}^{\infty}$ and the differential forms have constant rank. Sum over crossed repeated indices is understood. We will denote by $\mathcal{X}(M), \Omega^{p}(M)$ and $\mathrm{C}^{\infty}(M)$ the sets of vector fields, differentiable $p$-forms and 
functions in the manifold $M$ respectively. Finally $i(X) \alpha$ will denote the inner product or contraction of $X \in \mathcal{X}(M)$ with $\alpha \in \Omega^{p}(M)$ and $\mathrm{L}(X) \alpha$ the Lie derivative of the form $\alpha$ along the vector field $X$. Finally, along the work, quotient of manifolds by involutive distributions will be made; and then we assume that the corresponding quotient spaces are differentiable manifolds (conditions in order to assure this fact are stated in [2]).

\section{$2 \quad$ Presymplectic group actions}

\subsection{Presymplectic manifolds: previous statements}

Let us first recall that a presymplectic manifold is a couple $(M, \Omega)$ where $M$ is a $m$-dimensional differentiable manifold and $\Omega \in \Omega^{2}(M)$ is a closed degenerate differentiable form in $M$. Let

$$
\operatorname{ker} \Omega:=\{Z \in \mathcal{X}(M) \mid i(Z) \Omega=0\}
$$

which is assumed to be a distribution on $M$ (that is, it has constant rank).

A vector field $X \in \mathcal{X}(M)$ is said to be a Hamiltonian vector field (with respect to the presymplectic structure $\Omega$ ) iff $i(X) \Omega$ is an exact 1-form; that is, there exists $f_{X} \in \mathrm{C}^{\infty}(M)$ such that

$$
i(X) \Omega=\mathrm{d} f_{X}
$$

We will denote by $\mathcal{X}_{h}(M)$ the set of Hamiltonian vector fields in $M$.

$X \in \mathcal{X}(M)$ is said to be a locally Hamiltonian vector field (with respect to the presymplectic structure $\Omega$ ) iff $i(X) \Omega$ is a closed 1-form. In this case, for every point $x \in M$, there is an open neighbourhood $U \subset M$ and $f \in \mathrm{C}^{\infty}(U)$ such that

$$
\left.i(X) \Omega\right|_{U}=\mathrm{d} f
$$

We will denote by $\mathcal{X}_{l h}(M)$ the set of locally Hamiltonian vector fields in $M$, and it is obvious that $\mathcal{X}_{h}(M) \subset \mathcal{X}_{l h}(M)$. On the other hand, it is also immediate to observe that $X \in \mathcal{X}_{l h}(M)$ if, and only if, $\mathrm{L}(X) \Omega=0$. Finally, for every $X \in \mathcal{X}_{l h}(M)$ and $Z \in \operatorname{ker} \Omega$, we have that $[X, Z] \in \operatorname{ker} \Omega$.

$f \in \mathrm{C}^{\infty}(M)$ is said to be a presymplectic Hamiltonian function iff there exist a vector field $X \in \mathcal{X}(M)$ such that (11) holds. We will denote by $X_{f}$ the Hamiltonian vector field associated with $f$ and by $\mathrm{C}_{h}^{\infty}(M)$ the set of presymplectic Hamiltonian functions in $M$. If $f$ is a presymplectic Hamiltonian function then $\mathrm{L}(Z) f=0$, for every $Z \in$ ker $\Omega$ (and the same results holds for locally Hamiltonian functions in $U \subset M)$.

Since ker $\Omega \subset \mathcal{X}_{h}(M)$, then, if $X \in \mathcal{X}_{h}(M)$ and $Z \in \operatorname{ker} \Omega$, then $f_{X}=f_{X+Z}$ and, conversely, if $X, Y \in \mathcal{X}_{h}(M)$ and $f_{X}=f_{Y}$, therefore a vector field $Z \in$ ker $\Omega$ exists such that $X=Y+Z$. On the other hand, if $f \in \mathrm{C}_{h}^{\infty}(M)$ and $\lambda \in \mathbf{R}$ then $X_{f}=X_{f+\lambda}$ and, conversely, if $f, g \in \mathrm{C}_{h}^{\infty}(M)$ and $X_{f}=X_{g}$ then there exists $\lambda \in \mathbf{R}$ such that $f=g+\lambda$ (remember that $M$ is supposed to be connected).

Let $f_{1}, f_{2} \in \mathrm{C}_{h}^{\infty}(M)$ be presymplectic Hamiltonian functions and $X_{1}, X_{2} \in \mathcal{X}_{h}(M)$ Hamiltonian vector fields for these functions. The Poisson bracket of these Hamiltonian functions (related to the presymplectic structure $\Omega$ ) is the function $\left\{f_{1}, f_{2}\right\}$ given by

$$
\left\{f_{1}, f_{2}\right\}:=\Omega\left(X_{1}, X_{2}\right)=i\left(X_{2}\right) i\left(X_{1}\right) \Omega=i\left(X_{2}\right) \mathrm{d} f_{1}=-i\left(X_{1}\right) \mathrm{d} f_{2}
$$

It is trivial to prove that this definition does not depend on the Hamiltonian vector fields we have chosen. In addition, $\left\{f_{1}, f_{2}\right\} \in \mathrm{C}_{h}^{\infty}(M)$ and $i\left(\left[X_{1}, X_{2}\right]\right) \Omega=\mathrm{d}\left\{f_{2}, f_{1}\right\}$, in fact,

$$
i\left(\left[X_{1}, X_{2}\right]\right) \Omega=\mathrm{L}\left(X_{1}\right) i\left(X_{2}\right) \Omega-i\left(X_{1}\right) \mathrm{L}\left(X_{2}\right) \Omega=\mathrm{L}\left(X_{1}\right) i\left(X_{2}\right) \Omega=\mathrm{L}\left(X_{1}\right) \mathrm{d} f_{2}=\mathrm{d}\left\{f_{2}, f_{1}\right\}
$$


hence, $\mathcal{X}_{h}(M)$ is a Lie subalgebra of $\mathcal{X}(M)$. The same thing holds for $\mathcal{X}_{l h}(M)$ and ker $\Omega$ is an ideal of both algebras. So we have a map $\left(f_{1}, f_{2}\right) \mapsto\left\{f_{1}, f_{2}\right\}$ defined in $\mathrm{C}_{h}^{\infty}(M) / \mathbf{R}$ which transforms it into a real Lie algebra. In order to prove the Jacobi identity, observe that, from the last equality, we obtain that $\Omega\left(\left[X_{1}, X_{2}\right], X_{3}\right)=-\left\{f_{3},\left\{f_{2}, f_{1}\right\}\right\}$.

Considering the map $\Omega^{\sharp}: \mathcal{X}(M) \rightarrow \Omega^{1}(M)$ defined by $\Omega^{\sharp}(X):=i(X) \Omega$, for every $X \in \mathcal{X}(M)$, its restriction $\Omega_{h}^{\sharp}: \mathcal{X}_{h}(M) \rightarrow \mathrm{dC}_{h}^{\infty}(M)$ goes down to the quotient $\mathcal{X}_{h}(M) /$ ker $\Omega$, which is a Lie algebra because ker $\Omega$ is an ideal of the Lie algebra $\mathcal{X}_{h}(M)$, and hence the map $\tilde{\Omega}: \mathcal{X}_{h}(M) / \operatorname{ker} \Omega \rightarrow$ $\mathrm{C}_{h}^{\infty}(M) / \mathbf{R}$ is bijective and, according to the previous remark, a Lie algebra (anti) isomorphism.

\subsection{Actions of Lie groups on presymplectic manifolds}

Let $G$ be a Lie group (which we will assume to be connected), $\mathbf{g}$ its Lie algebra, $(M, \Omega)$ a presymplectic manifold and $\Phi: G \times M \rightarrow M$ a presymplectic action of $G$ on $M$; that is, $\Phi_{g}^{*} \Omega=\Omega$, for every $g \in G$. As a consequence, the fundamental vector field $\tilde{\xi} \in \mathcal{X}(M)$, associated with every $\xi \in \mathbf{g}$ by $\Phi$, is a locally Hamiltonian vector field, $\tilde{\xi} \in \mathcal{X}_{l h}(M)$ (conversely, if for every $\xi \in \mathbf{g}$, we have that $\tilde{\xi} \in \mathcal{X}_{l h}(M)$, then $\Phi$ is a presymplectic action of $G$ on $\left.M\right)$. In this case we have that, for every $\xi \in \mathbf{g}, \mathrm{L}(\tilde{\xi}) \Omega=0$ or, what is equivalent, $i(\tilde{\xi}) \Omega \in Z^{1}(M)$ (it is a closed 1-form). We denote by $\tilde{\mathbf{g}}$ the set of fundamental vector fields.

Now, following the same terminology as for actions of Lie groups on symplectic manifolds [1], [34], [46], [51, we state:

Definition $1 \Phi$ is said to be a strongly presymplectic or Hamiltonian action of $G$ on $M$ iff, $\tilde{\mathbf{g}} \subseteq \mathcal{X}_{h}(M)$ or, what is equivalent, for every $\xi \in \mathbf{g}, i(\tilde{\xi}) \Omega$ is an exact form. Otherwise, it is called $a$ weakly presymplectic or locally Hamiltonian action of $G$ on $M$.

It is important to discuss when a presymplectic action is strongly presymplectic. The fundamental obstruction appears because the map $\Omega^{\sharp}$ is not an isomorphism and, as a consequence, we have the following sequence of Lie algebras:

$$
0 \longrightarrow \operatorname{ker} \Omega \longrightarrow \mathcal{X}_{h}(M) \longrightarrow \mathcal{X}_{h}(M) / \operatorname{ker} \Omega \longrightarrow 0
$$

but $\mathcal{X}_{h}(M) /$ ker $\Omega \simeq \Omega^{\sharp}\left(\mathcal{X}_{h}(M)\right)$, then denoting $\mathcal{X}_{h}(M) /$ ker $\Omega \equiv B_{h}^{1}(M)$, we have that $B_{h}^{1}(M) \subset$ $B^{1}(M)$ (where $B^{1}(M)$ is the set of exact differential 1-forms in $M$ ) and it is a strict inclusion. In an analogous way we have the sequence

$$
0 \longrightarrow \operatorname{ker} \Omega \longrightarrow \mathcal{X}_{l h}(M) \longrightarrow \mathcal{X}_{l h}(M) / \operatorname{ker} \Omega \longrightarrow 0
$$

but $\mathcal{X}_{l h}(M) /$ ker $\Omega \simeq \Omega^{\sharp}\left(\mathcal{X}_{l h}(M)\right)$, then denoting $\mathcal{X}_{l h}(M) /$ ker $\Omega \equiv Z_{h}^{1}(M)$, we have that $Z_{h}^{1}(M) \subset$ $Z^{1}(M)$ (where $Z^{1}(M)$ are the closed differential 1-forms in $M$ ), and this is also a strict inclusion. There is no problem with these exact sequences and the morphisms relating them, but it is not possible to identify $\mathcal{X}_{l h}(M) / \mathcal{X}_{h}(M)$ with $H^{1}(M)$ (the first de Rham's cohomology group of $M$ ), like in the symplectic case. Nevertheless, we have

$$
\mathcal{X}_{l h}(M) / \mathcal{X}_{h}(M) \simeq\left(\mathcal{X}_{l h}(M) / \operatorname{ker} \Omega\right) /\left(\mathcal{X}_{h}(M) / \operatorname{ker} \Omega\right) \simeq Z_{h}^{1}(M) / B_{h}^{1}(M)
$$

and we can write

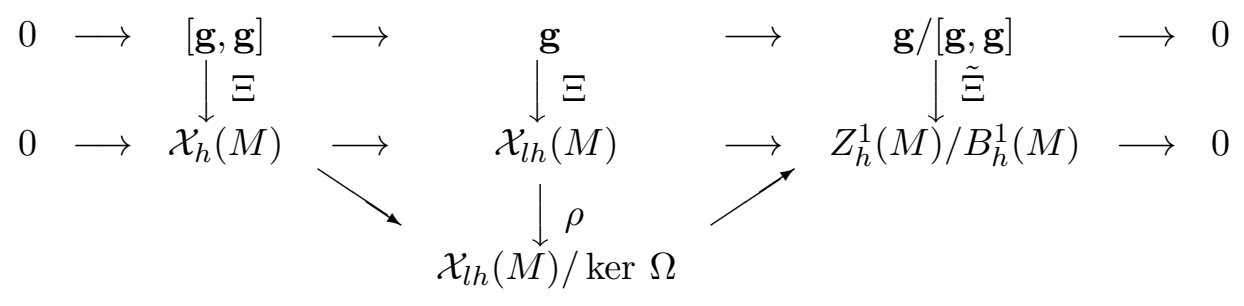


where $\tilde{\Xi}$ is a Lie algebra homomorphism which makes the diagram commutative. Then, the action is strongly presymplectic (that is, the image of $\mathbf{g}$ by $\Xi$ is in $\mathcal{X}_{h}(M)$ ) if and only if $\tilde{\Xi}=0$. Obviously, if $H^{1}(M)=0$, then $Z^{1}(M)=B^{1}(M)$ and $\mathcal{X}_{h}(M)=\mathcal{X}_{l h}(M)$, therefore $Z_{h}^{1}(M)=B_{h}^{1}(M)$.

In particular, if $(M, \Omega)$ is an exact presymplectic manifold (that is, there exists $\Theta \in \Omega^{1}(M)$ such that $\mathrm{d} \Theta=\Omega$ ) and $\Phi$ is an exact action (that is, $\Phi_{g}^{*} \Theta=\Theta$, for every $g \in G$ ) then $\Phi$ is strongly presymplectic.

\subsection{Momentum mapping}

Let $G$ be a Lie group, $(M, \Omega)$ a presymplectic manifold and $\Phi: G \times M \rightarrow M$ a presymplectic action of $G$ on $M$.

Definition 2 1. A comomentum mapping associated with $\Phi$ [50 is a Lie algebra map (if it exists)

$$
\begin{aligned}
& \mathcal{J}^{*}: \mathbf{g} \rightarrow \mathrm{C}_{h}^{\infty}(M) \\
& \xi \mapsto f_{\xi}
\end{aligned}
$$

such that $i(\tilde{\xi}) \Omega=\mathrm{d} f_{\xi}$; or, what is equivalent, such that the following diagram commutes

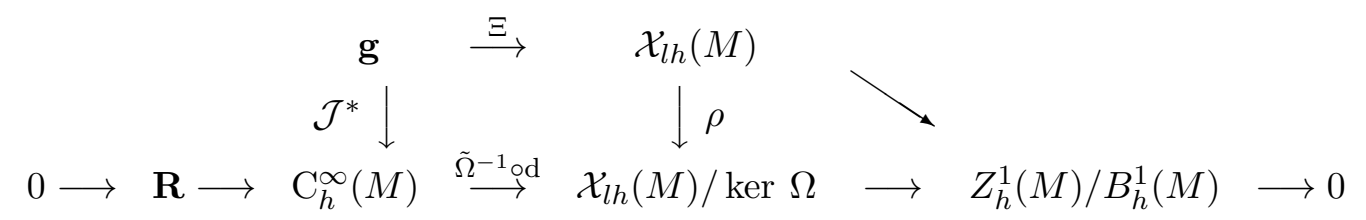

2. A momentum mapping associated with $\Phi$ is the dual map of a comomentum mapping; in other words, it is a map $\mathcal{J}: M \rightarrow \mathbf{g}^{*}$ such that, for every $\xi \in \mathbf{g}$ and $x \in M$,

$$
(\mathcal{J}(x))(\xi):=\mathcal{J}^{*}(\xi)(x)=f_{\xi}(x)
$$

As in the symplectic case we have:

Proposition 1 A comomentum map and the dual momentum map associated with the presymplectic action $\Phi$ on $(M, \Omega)$ exist if, and only if, the action is strongly presymplectic.

( Proof) In fact; by definition, if a comomentum mapping exists, then $\tilde{\Omega}^{-1} \circ \mathrm{d} \circ \mathcal{J}^{*}=\rho \circ \Xi$ (see (3) $)$, but then $\operatorname{Im}(\rho \circ \Xi) \subset \operatorname{Im} \tilde{\Omega}^{-1}=\mathcal{X}_{h}(M) / \operatorname{ker} \Omega$, and this implies that $\operatorname{Im} \Xi \subset \mathcal{X}_{h}(M)$ and the action is strongly presymplectic.

Conversely, if the action is strongly presymplectic: $\operatorname{Im} \Xi \subset \mathcal{X}_{h}(M)$, then we have that ( $\rho \circ$ $\Xi)(\mathbf{g}) \subset \mathcal{X}_{h}(\underset{\tilde{\xi}}{M}) /$ ker $\Omega$ and then, for all $\xi \in \mathbf{g}$, there exists a unique (except constants) $f_{\xi} \in \mathrm{C}_{h}^{\infty}(M)$ such that $i(\tilde{\xi}) \Omega=\mathrm{d} f_{\xi}$, and this is a Lie algebra homomorphism.

Therefore, if a comomentum mapping exists, the commutative part of the diagram (3) reduces to

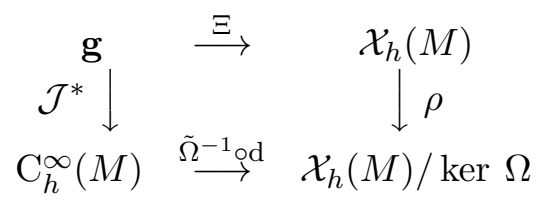


As in the symplectic case, it is important to point out that, if a comomentum map $\mathcal{J}^{*}$ exists for a presymplectic action, and $\mathcal{F}: \mathbf{g} \rightarrow \mathbf{R}$ is a linear map (that is, $\mathcal{F} \in \mathbf{g}^{*}$ ), then $\mathcal{J}^{*}+\mathcal{F}$ is another comomentum map for the same action $\Phi$. Moreover, a comomentum map is not necessarily a Lie algebra homomorphism. Then:

Definition 3 The action $\Phi$ is said to be a Poissonian or strongly Hamiltonian action iff:

1. There exists a comomentum mapping for this action (and then also a momentum one).

2. It is a Lie algebra homomorphism.

As a particular case, we have that, if $(M, \Omega)$ is an exact presymplectic manifold with $\Omega=\mathrm{d} \Theta$, and the action $\Phi$ of $G$ on $M$ is exact, then:

1. A momentum mapping exists and it is given by $\mathcal{J}(\xi)=-\Theta(\tilde{\xi})=-i(\tilde{\xi}) \Theta$, for every $\xi \in \mathbf{g}$.

2. The action is Poissonian.

In fact, the first item is immediate. For the second one we have

$$
f_{\left[\xi_{1}, \xi_{2}\right]}=-i\left(\left[\tilde{\xi}_{1}, \tilde{\xi}_{2}\right]\right) \Theta=-\mathrm{L}\left(\tilde{\xi}_{1}\right) i\left(\tilde{\xi}_{2}\right) \Theta=\mathrm{L}\left(\tilde{\xi}_{1}\right) f_{\xi_{2}}=\left\{f_{\xi_{1}}, f_{\xi_{2}}\right\}
$$

In other cases, local comomentum mappings can always be defined for every presymplectic action, although without necessarily being Lie algebra homomorphisms.

In addition, we have that if $G$ is a connected Lie group and $\Phi$ is a strongly presymplectic action of $G$ on the presymplectic manifold $(M, \Omega)$. Then the following statements are equivalent:

1. The momentum mapping associated with this action is $A d^{*}$-equivariant, that is, for every $g \in G$, the following diagram commutes:

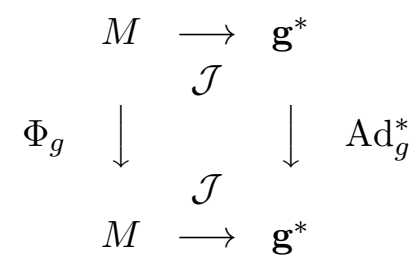

2. The action is Poissonian.

(The proof of this statement is the same as for the symplectic case and can be found in any of the above mentioned references).

\subsection{Level sets of the momentum mapping}

First remember that, if $\Phi$ is a strongly presymplectic action of a Lie group $G$ on $(M, \Omega)$ and $\mathcal{J}$ is the momentum mapping associated to this action, then $\mu \in \mathbf{g}^{*}$ is a weakly regular value of $\mathcal{J}$ iff

1. $\mathcal{J}^{-1}(\mu)$ is a submanifold of $M$.

2. $\mathrm{T}_{x}\left(\mathcal{J}^{-1}(\mu)\right)=\operatorname{ker} \mathrm{T}_{x} \mathcal{J}$, for every $x \in \mathcal{J}^{-1}(\mu)$. 
If $\mathrm{T}_{x} \mathcal{J}$ is surjective, $\mu$ is said to be a regular value. Of course, every regular value is weakly regular.

Taking into account that if a fundamental vector field belongs to ker $\Omega$ its Hamiltonian function can be taken to be zero, we have that:

Proposition 2 If $\mu$ is a weakly regular value of $\mathcal{J}$ then $\mu(\xi)=0$, for every $\xi \in \mathbf{g}$ such that $\tilde{\xi} \in \tilde{\mathbf{g}} \cap \operatorname{ker} \Omega$.

From now on we will assume $\mu \in \mathbf{g}^{*}$ is, at least, a weakly regular value of $\mathcal{J}$. So, we will denote by $j_{\mu}: \mathcal{J}^{-1}(\mu) \hookrightarrow M$ the corresponding imbedding. Then, in order to make a description of $\mathcal{J}^{-1}(\mu)$, we have that the constraints defining it are the component functions of $\mathcal{J}=\mu$. In fact, observe that, if $\left\{\xi_{i}\right\}$ is a basis of $\mathbf{g},\left\{f_{\xi_{i}}\right\}$ are the Hamiltonian functions associated to the fundamental vector fields $\left\{\tilde{\xi}_{i}\right\}$ by the comomentum map and $\left\{\alpha^{i}\right\}$ is the dual basis in $\mathbf{g}^{*}$, then $\mu=\mu_{i} \alpha^{i}$, with $\mu_{i}$ real numbers, and we have that

$$
\begin{aligned}
\mathcal{J}^{-1}(\mu) & :=\{x \in M \mid \mathcal{J}(x)=\mu\}=\{x \in M \mid(\mathcal{J}(x))(\xi)=\mu(\xi), \forall \xi \in \mathbf{g}\} \\
& =\left\{x \in M \mid(\mathcal{J}(x))\left(\xi_{i}\right)=\mu_{i}\right\}=\left\{x \in M \mid f_{\xi_{i}}(x)=\mu_{i}\right\}
\end{aligned}
$$

that is, $j_{\mu}^{*} f_{\xi_{i}}-\mu_{i}=0$, and then the constraints are $\zeta_{i}:=f_{\xi_{i}}-\mu_{i}$. Notice that this is equivalent to saying that the expression of the momentum mapping is

$$
\mathcal{J}(x) \equiv f_{\xi_{i}}(x) \alpha^{i}
$$

Bearing in mind a well known result in the theory of exterior differential systems (see, for instance, [ $[$ ]), we have that all the level sets of the momentum mapping can also be obtained as the integral submanifolds of a Pfaff system. In fact:

Proposition 3 Let $G$ be a Lie group and $\Phi$ a strongly presymplectic action of $G$ on the presymplectic manifold $(M, \Omega)$. The connected components of the level sets of the momentum mapping $\mathcal{J}$ associated with this action are the connected maximal integral submanifolds of the Pfaff system $i(\tilde{\xi}) \Omega=0$, for $\tilde{\xi} \in \tilde{\mathbf{g}}$.

As a consequence of this proposition, we obtain that:

Corollary 1 If $x \in \mathcal{J}^{-1}(\mu)$ then $\mathrm{T}_{x} \mathcal{J}^{-1}(\mu)=\tilde{\mathbf{g}}_{x}^{\perp}$. As a consequence, since ker $\Omega_{x} \subset \tilde{\mathbf{g}}_{x}^{\perp}$, then ker $\Omega \subset \underline{\mathcal{X}\left(\mathcal{J}^{-1}(\mu)\right)}$ (where $\underline{\mathcal{X}\left(\mathcal{J}^{-1}(\mu)\right)}$ denotes the set of vector fields of $\mathcal{X}(M)$ which are tangent to $\left.\mathcal{J}^{-1}(\mu)\right)$.

If $\Omega=\mathrm{d} \Theta$ and the action is exact, then $f_{\xi}=-i(\tilde{\xi}) \Theta$ and Pfaff system $i(\tilde{\xi}) \Omega=0$ can be equivalently expressed as $\mathrm{d} i(\tilde{\xi}) \Theta=0$.

From now on, we will assume the following:

Assumption 1 The action $\Phi$ that we will consider will be Poissonian, free and proper and $\mu$ will be a weakly regular value of the momentum mapping associated to this action.

Let $G_{\mu}$ be the isotropy group of $\mu$ for the coadjoint action of $G$ on $\mathbf{g}^{*}$. Then we have: 
Theorem $1 G_{\mu}$ is the maximal subgroup of $G$ which lets $\mathcal{J}^{-1}(\mu)$ invariant and so the quotient $\mathcal{J}^{-1}(\mu) / G_{\mu}$ is well defined and it is called the reduced phase space or the orbit space of $\mathcal{J}^{-1}(\mu)$.

( Proof) For every $x \in M$ such that $\mathcal{J}(x)=\mu$ and $g \in G_{\mu}$, we have

$$
\mathcal{J}\left(\Phi_{g}(x)\right)=\left(\mathcal{J} \circ \Phi_{g}\right)(x)=\left(\operatorname{Ad}_{g}^{*} \circ \mathcal{J}\right)(x)=\operatorname{Ad}_{g}^{*}(\mu)=\mu
$$

then $\Phi_{g}(x) \in \mathcal{J}^{-1}(\mu)$, so $\mathcal{J}^{-1}(\mu)$ is invariant under the action of $G_{\mu}$ and the quotient is well defined.

The maximality of $G_{\mu}$ is a direct consequence of the equivariance of $\mathcal{J}$.

If $\mathbf{g}_{\mu}$ is the Lie algebra of $G_{\mu}$ then, as a consequence of this theorem, $\tilde{\mathbf{g}}_{\mu}$ are vector fields tangent to $\mathcal{J}^{-1}(\mu)$, and we have that $\tilde{\mathbf{g}}_{\mu}=\tilde{\mathbf{g}} \cap \underline{\mathcal{X}\left(\mathcal{J}^{-1}(\mu)\right)}$.

At this point, it is interesting to point out two different possibilities:

- $\tilde{\mathbf{g}} \cap \operatorname{ker} \Omega=\{0\}$ : In this case all the fundamental vector fields give constraints which are not constant functions. Then $\operatorname{dim} \mathcal{J}^{-1}(\mu)<m=\operatorname{dim} M$.

- $\tilde{\mathbf{g}} \cap \operatorname{ker} \Omega \neq\{0\}$ : Now, only those fundamental vector fields such that $\tilde{\xi} \notin$ ker $\Omega$ give constraints which are not constant functions. Then $\operatorname{dim} \mathcal{J}^{-1}(\mu) \leq m$.

$\mathcal{J}^{-1}(\mu)$ inherits a presymplectic structure $\Omega_{\mu}:=j_{\mu}^{*} \Omega$. We are going to characterize ker $\Omega_{\mu}$. First of all we have that $\tilde{\mathbf{g}}_{\mu} \subset \mathcal{X}\left(\mathcal{J}^{-1}(\mu)\right)$ (and hence, for every $\tilde{\xi} \in \tilde{\mathbf{g}}_{\mu}$, there exists $\tilde{\xi}_{\mu} \in \mathcal{X}\left(\mathcal{J}^{-1}(\mu)\right.$ ) such that $\left.j_{\mu *} \tilde{\xi}_{\mu}=\left.\tilde{\xi}\right|_{\mathcal{J}^{-1}(\mu)}\right)$. Consider now the orthogonal presymplectic complement of $\underline{\mathcal{X}\left(\mathcal{J}^{-1}(\mu)\right)}$ in $\mathcal{X}(M)$, that is, the set

$$
\begin{aligned}
\left(\mathcal{X}\left(\mathcal{J}^{-1}(\mu)\right)\right)^{\perp} & :=\left\{Z \in \mathcal{X}(M) \mid(i(X) i(Z) \Omega)(x)=0, \forall X \in \underline{\mathcal{X}\left(\mathcal{J}^{-1}(\mu)\right)}, \forall x \in \mathcal{J}^{-1}(\mu)\right\} \\
& =\left\{Z \in \mathcal{X}(M) \mid j_{\mu}^{*} i(Z) \Omega=0\right\}
\end{aligned}
$$

Then, let

$$
\operatorname{ker} \Omega_{\mu}:=\left\{Y_{\mu} \in \mathcal{X}\left(\mathcal{J}^{-1}(\mu)\right) \mid i\left(Y_{\mu}\right) \Omega_{\mu}=0\right\}
$$

and denoting by ker $\Omega_{\mu}$ the set of vector fields of $\mathcal{X}(M)$ such that $\left.\operatorname{ker} \Omega_{\mu}\right|_{\mathcal{J}^{-1}(\mu)}=j_{\mu *}$ ker $\Omega_{\mu}$, it is immediate to prove that

$$
\underline{\operatorname{ker} \Omega_{\mu}}=\underline{\mathcal{X}\left(\mathcal{J}^{-1}(\mu)\right)} \cap\left(\mathcal{X}\left(\mathcal{J}^{-1}(\mu)\right)\right)^{\perp}
$$

Therefore, we have the following result:

Proposition 4 ker $\Omega_{\mu_{x}}=\tilde{\mathbf{g}}_{\mu_{x}}+$ ker $\Omega_{x}$, for every $x \in \mathcal{J}^{-1}(\mu)$.

( Proof) For the proof see the appendix with the following identifications: $E=\mathrm{T}_{x} M, S=\tilde{\mathbf{g}}_{x}$, $N=S^{\perp}=\tilde{\mathbf{g}}_{x}^{\perp}=\mathrm{T}_{x} \mathcal{J}^{-1}(\mu)$, and $S \cap N=\tilde{\mathbf{g}}_{x} \cap \mathrm{T}_{x} \mathcal{J}^{-1}(\mu)=\tilde{\mathbf{g}}_{\mu_{x}}$.

At this point, we can state the following result which generalizes the idea of the MarsdenWeinstein reduction theorem [42], [52] to presymplectic actions of Lie groups on presymplectic manifolds:

Theorem 2 The orbit space $\mathcal{J}^{-1}(\mu) / G_{\mu}$ is a differentiable manifold. Then, if $\sigma: \mathcal{J}^{-1}(\mu) \rightarrow$ $\mathcal{J}^{-1}(\mu) / G_{\mu}$ denotes the canonical projection, there is a closed 2-form $\hat{\Omega} \in \Omega^{2}\left(\mathcal{J}^{-1}(\mu) / G_{\mu}\right)$ such that $\Omega_{\mu}=\sigma^{*} \hat{\Omega}$ (that is, $\Omega_{\mu}$ is $\sigma$-projectable), and: 
- $\hat{\Omega}$ is symplectic if, and only if, for every $x \in \mathcal{J}^{-1}(\mu), \tilde{\mathbf{g}}_{\mu_{x}}=\operatorname{ker} \Omega_{\mu_{x}}$ or, what is equivalent, ker $\Omega_{x} \cap \mathrm{T}_{x} \mathcal{J}^{-1}(\mu) \subseteq \tilde{\mathrm{g}}_{\mu_{x}}$.

- Otherwise $\hat{\Omega}$ is presymplectic. In particular, for every $x \in \mathcal{J}^{-1}(\mu)$, if $\operatorname{ker} \Omega_{x} \subset \mathrm{T}_{x} \mathcal{J}^{-1}(\mu)$ and $\tilde{\mathbf{g}}_{x} \cap \operatorname{ker} \Omega_{x}=\{0\}$, then $\operatorname{rank} \hat{\Omega}=\operatorname{rank} \Omega$.

( Proof ) For the proof of the first part of this statement $\left(\mathcal{J}^{-1}(\mu) / G_{\mu}\right.$ is a differentiable manifold) see [1], 42] or [34]. The existence of $\hat{\Omega}$ and the two items of the second part are a direct consequence of proposition 4 .

\section{Symmetries of presymplectic dynamical systems and reduction}

\subsection{Review on presymplectic dynamical systems}

One of the most important features in the study of dynamical systems with symmetry is the socalled reduction theory. Next we want to apply the above results in order to state the main results on this topic concerning presymplectic dynamical systems, generalizing the ideas of the MarsdenWeinstein symplectic reduction procedure [42], [52].

We start by giving the background ideas on presymplectic dynamical systems. For further information on this topic one may see, for instance, [23], [14], [7], 433], [13], [25] (see also [19] as a pioneering work).

A presymplectic locally Hamiltonian dynamical system is a triad $(P, \omega, \alpha)$, where $(P, \omega)$ is a presymplectic manifold and $\alpha \in Z^{1}(P)$. If $\alpha$ is exact then $\alpha=\mathrm{d} \mathcal{H}$ for some $\mathcal{H} \in \mathrm{C}^{\infty}(P)$, and then the triad $(P, \omega, \mathcal{H})$ is said to be a presymplectic Hamiltonian system (and this is the case we are going to consider, without loss of generality).

Every presymplectic dynamical system has associated the following equation

$$
i\left(X_{P}\right) \omega=\mathrm{d} \mathcal{H} \quad ; \quad X_{P} \in \mathcal{X}(P)
$$

which is compatible everywhere in $P$ if, and only if, $i(Z) \mathrm{d} \mathcal{H}=0$, for every $Z \in$ ker $\omega$. In this case $X_{P} \in \mathcal{X}_{h}(P)$; and the presymplectic dynamical system is said to be compatible. If the equation is not compatible, in the most interesting cases, there is a (maximal) closed regular submanifold $j_{M}: M \hookrightarrow P$, for which a vector field $X_{P}$ tangent to $M$ exists such that the following equation holds

$$
\left.\left[i\left(X_{P}\right) \omega-\mathrm{d} \mathcal{H}\right]\right|_{M}=0
$$

(and this is an equation for $X_{P}$ and $M$ ). $M$ is called the final constraint submanifold and inherits a presymplectic structure $\Omega=j_{M}^{*} \omega$. This submanifold is obtained at the end of a recursive algorithm which gives a sequence of submanifolds

$$
P \hookleftarrow P_{1} \hookleftarrow \ldots \hookleftarrow P_{i-1} \hookleftarrow P_{i} \hookleftarrow \ldots \hookleftarrow P_{f-1} \hookleftarrow P_{f} \equiv M
$$

The equation (6) can be pulled-back to $M$ obtaining

$$
j_{M}^{*}\left[i\left(X_{P}\right) \omega-\mathrm{d} \mathcal{H}\right]=i(X) \Omega-\mathrm{dH}=0
$$

with $\mathrm{H}=j_{M}^{*} \mathcal{H}, X_{P} \in \mathcal{X}(P)$ tangent to $M, X \in \mathcal{X}(M)$ and $j_{M_{*}} X=\left.X_{P}\right|_{M}$. Notice that this is a compatible system because if $X_{P} \in \mathcal{X}(P)$ tangent to $M$ is a vector field solution of (6), this implies that the equation (]) holds for $X$. 
For the final submanifold $M$ the vector field $X_{P}$ satisfying (6) is not unique, in general. Then the difference between two solutions is called a gauge vector field; and the points of $M$ reached from another fixed one $x \in M$ by means of an integral curve of a gauge vector field (passing through $x$ ) are the so-called gauge equivalent points or states. Under certain regularity conditions, it is proved that the set of gauge vector fields is just $\underline{\operatorname{ker} \Omega}$ (the necessary and sufficient condition for this is the following [22], [7]: the constraint functions locally defining $M$ in $P$ can be classified into first and second class; then there is a basis of the set of first class constraints whose differentials do not vanish along $M)$.

In order to remove the redundancy of solutions, it is assumed that gauge equivalent states represent the same physical state. Geometrically this means that we must go from $M$ to the quotient of $M$ by the foliation generated by the involutive distribution ker $\Omega$. It is assumed that the quotient space $\bar{M} \equiv M / \operatorname{ker} \Omega$ is a differentiable manifold, the projection $\pi_{M}: M \rightarrow \bar{M}$ is a submersion and $\bar{M}$ is endowed with a symplectic structure $\bar{\omega}$ such that $\pi_{M}^{*} \bar{\omega}=\Omega .(\bar{M}, \bar{\omega})$ is called the manifold of real physical states, and the equations (16) and (耳) project in a natural way to $\bar{M}$ :

$$
i\left(\bar{X}_{P}\right) \bar{\omega}-\mathrm{d} \overline{\mathrm{H}}=0
$$

where $\pi_{M}^{*} \overline{\mathrm{H}}=\mathrm{H}$ and for every $X_{P} \in \mathcal{X}(M)$ which is solution of (6), there is a $\pi_{M}$-projectable vector field $X \in \mathcal{X}(M)$, with $j_{M_{*}} X=\overline{\left.X_{P}\right|_{M}}$, such that $\pi_{M_{*}} X=\bar{X}_{P}$ is the unique solution of (8). Note that the existence of $\overline{\mathrm{H}}$ is assured because $i(\operatorname{ker} \Omega) \mathrm{H}=0$, since the dynamical equation (77) on $M$ is compatible. This is the so-called gauge reduction procedure.

On the other hand, the following structure theorem for presymplectic dynamical systems plays a crucial role in some of the developments of this work:

Theorem 3 Let $(M, \Omega, \mathrm{H})$ be a compatible presymplectic dynamical system. Then:

1. There exists a symplectic manifold $(\mathbf{M}, \mathbf{\Omega})$ such that $j_{0}: M \hookrightarrow \mathbf{M}$ is a coisotropic imbedding, and $j_{0}^{*} \boldsymbol{\Omega}=\Omega$.

2. There exists a family $\mathcal{D}_{l h}(\mathbf{M}, M)$ of symplectic locally Hamiltonian vector fields in $\mathbf{M}$ tangent to $M$, which gives all the dynamical solutions of the equation

$$
j_{0}^{*}\left[i\left(X_{\beta}\right) \boldsymbol{\Omega}-\mathrm{d} \mathrm{H}\right]=i(X) \Omega-\mathrm{d} \mathrm{H}=0 \quad ; \quad X_{\beta} \in \mathcal{D}_{l h}(\mathbf{M}, M)
$$

3. The symplectic manifold $(\mathbf{M}, \boldsymbol{\Omega})$ and the family $\mathcal{D}_{l h}(\mathbf{M}, M)$ are unique up to symplectomorphic neighbourhood equivalences between symplectic manifolds containing $(M, \Omega)$ as a coisotropic submanifold; and all these symplectomorphisms reduce to the identity on $M$. (That is, if $j_{i}: M \hookrightarrow\left(\mathbf{M}_{i}, \boldsymbol{\Omega}_{i}\right), i=1,2$, are two coisotropic imbeddings, then there exist two tubular neighbourhoods $U_{i}$ of $j_{i}(M)$ in $\mathbf{M}_{i}$ and a symplectomorphism $\psi:\left(\mathbf{M}_{1}, \boldsymbol{\Omega}_{1}\right) \rightarrow\left(\mathbf{M}_{2}, \boldsymbol{\Omega}_{2}\right)$ such that $\psi \circ j_{1}=j_{2}$ and $\psi_{*}\left(\mathcal{D}_{l h}\left(\mathbf{M}_{1}, M\right)=\mathcal{D}_{l h}\left(\mathbf{M}_{2}, M\right)\right)$.

The pair $(\mathbf{M}, \mathbf{\Omega})$ is called an ambient symplectic manifold for $(M, \Omega)$ and $(\mathbf{M}, \boldsymbol{\Omega}, \mathbf{H})$ is called an ambient symplectic dynamical system for the presymplectic system $(M, \Omega, \mathrm{H})$.

(Outline of the proof) The first part of this statement (together with the symplectomorphic equivalence of coisotropic imbeddings) is the well-known coisotropic imbedding theorem [24] [35]. The symplectic manifold $(\mathbf{M}, \boldsymbol{\Omega})$ is constructed as a tubular neighbourhood of the zero section of the dual characteristic bundle $K^{*} \equiv(\operatorname{ker} \Omega)^{*}$, which is identified with $M$. The strategy consists in considering the vector bundle $\pi_{K}: K \rightarrow M$; then, as $K$ is a subbundle of T $M$, using a metric 
in $M$, we can split $\mathrm{T} M=G \oplus K$, and then $\mathrm{T}_{M} K^{*}=\mathrm{T} M \oplus K^{*}=G \oplus K \oplus K^{*}$. Denoting $\sigma: \mathrm{T}_{M} K \rightarrow K \oplus K^{*}$, we set $\boldsymbol{\Omega}=\pi_{K}^{*} \Omega+\sigma^{*} \Omega_{K}$; where $\Omega_{K}$ is the symplectic form canonically defined in $K \oplus K^{*}$. Then $\Omega$ can be extended to a tubular neighbourhood of $M$ in $K^{*}$ using Weinstein's extension theorem [52].

In relation to the second part, the family $\mathcal{D}_{l h}(\mathbf{M}, M)$ is made of the vector fields $X_{\beta}=\mathbf{\Omega}^{-1}(\mathrm{~d} \mathbf{H}+$ $\beta$ ), where $\mathbf{H} \in \mathrm{C}^{\infty}(\mathbf{M})$ is an extension of $\mathrm{H}$ to $\mathbf{M}$ (that is, $\left.j_{0}^{*} \mathbf{H}=\mathrm{H}\right)$ and $\beta \in Z^{1}(\mathbf{M})$ is a closed first class constraint one form, (that is, $j_{0}^{*} \beta=0$ and $j_{0}^{*} i(Z) \beta=0, \forall Z \in \mathcal{X}(M)^{\perp}$ ). (See [14] for the details of this part of the proof).

Finally, the local uniqueness of the coisotropic imbedding is a straightforward consequence of the local uniqueness part of Weinstein's extension theorem.

From now on we will consider presymplectic dynamical systems $(P, \omega, \mathcal{H})$ with final constraint submanifold $(M, \Omega, \mathrm{H})$ which hold all these features.

\subsection{Reduction of compatible presymplectic dynamical systems with symmetry}

Consider now a compatible presymplectic dynamical system $(M, \Omega, \mathrm{H})$; that is, such that the dynamical equation

$$
i(X) \Omega-\mathrm{dH}=0
$$

has solution $X \in \mathcal{X}(M)$ everywhere in $M$. We are then able to introduce the concept of group of symmetries for a compatible presymplectic dynamical system (and its reduction) as follows (the case of non-compatible systems will be studied afterwards):

Definition 4 Let $G$ be a Lie group, $(M, \Omega, \mathrm{H})$ a compatible presymplectic dynamical system and $\Phi: G \times M \rightarrow M$ an action of $G$ on $M . G$ is said to be a symmetry group of this system iff

1. $\Phi$ is a presymplectic action on $(M, \Omega)$

2. $\Phi_{g}^{*} \mathrm{H}=\mathrm{H}$; for every $g \in G$ or, what is equivalent, $\mathrm{L}(\tilde{\xi}) \mathrm{H}=0$, for every $\xi \in \mathbf{g}$.

The diffeomorphism $\Phi_{g}$, for every $g \in G$, is called a symmetry of the system. The fundamental vector fields $\tilde{\xi} \in \tilde{\mathbf{g}}$ are the so-called infinitesimal generators of symmetries.

Obviously this definition is the same as the usual one for symmetries of symplectic dynamical systems. At this point, we first prove that gauge symmetries are symmetries of the presymplectic dynamical system.

Proposition 5 Let $(M, \Omega, \mathrm{H})$ be a compatible presymplectic dynamical system. Then the vector fields of $\operatorname{ker} \Omega$ are infinitesimal generators of symmetries of this system.

( Proof) First, for every $Z \in$ ker $\Omega$, by definition $i(Z) \Omega=0$ and hence $\mathrm{L}(Z) \Omega=0$. On the other hand, since the equation (9) is compatible, it implies that

$$
i(Z) \mathrm{dH}=\mathrm{L}(Z) \mathrm{H}=0
$$


According to the terminology of the above section, we say that the vector fields of ker $\Omega$ are infinitesimal generators of symmetries of the system. Then, if we want to remove the symmetries, following a reduction procedure in order to get a symplectic dynamical system, we must suppose that the vector fields in ker $\Omega$ are contained in the distribution generated by $\tilde{\mathbf{g}}$. So, from now on we will assume that:

Assumption $\mathbf{2}$ Let $\tilde{\mathbf{g}}$ be the vector space made of the fundamental vector fields of the action $\Phi$ of the symmetry group $G$ on $M$. Then $\operatorname{ker} \Omega \subset \mathrm{C}^{\infty}(M) \otimes \tilde{\mathbf{g}}$.

\section{Comments:}

- This assumption means that, if $\left\{\xi_{1}, \ldots, \xi_{h}\right\} \subset \mathbf{g}$ is a basis of $\mathbf{g}$, and $Z \in$ ker $\Omega$; then there exist $\left\{f^{i^{1}}, \ldots, f^{i^{h}}\right\} \subset \mathrm{C}^{\infty}(M)$ such that $Z=f^{i} \tilde{\xi}_{i}$.

- Observe that $\mathrm{C}^{\infty}(M) \otimes \tilde{\mathbf{g}}$ is the submodule of $\mathcal{X}(M)$ made of the vector fields tangent to the orbits of the action of $G$ (or, what is equivalent, if the distribution defined by $\tilde{\mathbf{g}}$ has constant dimension, they are the sections of this distribution). Therefore, the assumption means that the elements of ker $\Omega$ are tangent to these orbits. Hence, the leaves of the foliation induced by ker $\Omega$ are contained in those orbits.

- Notice that the elements of ker $\Omega$ are infinitesimal generators of symmetries but, in general, those of $\mathrm{C}^{\infty}(M) \otimes \tilde{\mathbf{g}}$ are not.

In the usual physical terminology, the vector fields of ker $\Omega$ are called infinitesimal generators of gauge symmetries. On the contrary, the vector fields of $\tilde{\mathbf{g}}$ which do not belong to ker $\Omega$ are the so-called infinitesimal generators of non-gauge or rigid symmetries.

Now, suppose that the action of the symmetry group $G$ on the compatible presymplectic dynamical system $(M, \Omega, \mathrm{H})$ is Poissonian. Let $\mathcal{J}$ be the momentum mapping associated with this action, and $\mu \in \mathbf{g}^{*}$ a weakly regular value of $\mathcal{J}$. Then the submanifold $\mathcal{J}^{-1}(\mu)$, the form $\Omega_{\mu}=j_{\mu}^{*} \Omega$ and the function $\mathrm{H}_{\mu}=j_{\mu}^{*} \mathrm{H}$ make a presymplectic Hamiltonian system $\left(\mathcal{J}^{-1}(\mu), \Omega_{\mu}, \mathrm{H}_{\mu}\right)$. Then we have:

Proposition 6 If $X \in \mathcal{X}_{h}(M)$ is a vector field solution of the equation (9), then:

1. $X$ is tangent to $\mathcal{J}^{-1}(\mu)$.

2. The dynamical equation

$$
i\left(X_{\mu}\right) \Omega_{\mu}-\mathrm{dH}_{\mu}=0
$$

is compatible and its solutions are $X_{\mu}+\operatorname{ker} \Omega_{\mu}$, where $X_{\mu} \in \mathcal{X}\left(\mathcal{J}^{-1}(\mu)\right)$ is a vector field such that $j_{\mu *} X_{\mu}=\left.X\right|_{\mathcal{J}^{-1}(\mu)}$.

( Proof) First we prove that if $X \in \mathcal{X}(M)$ is a solution of the equation (7), then $X \in \underline{\mathcal{X}\left(\mathcal{J}^{-1}(\mu)\right)}$. In fact, for every constraint $\zeta$, with $\mathrm{d} \zeta=i(\tilde{\xi}) \Omega, \tilde{\xi} \in \tilde{\mathbf{g}}$, defining $\mathcal{J}^{-1}(\mu)$,

$$
j_{\mu}^{*} X(\zeta)=j_{\mu}^{*}(i(X) \mathrm{d} \zeta)=j_{\mu}^{*}(i(X) i(\tilde{\xi}) \Omega)=-j_{\mu}^{*}(i(\tilde{\xi}) i(X) \Omega)=-j_{\mu}^{*}(i(\tilde{\xi}) \mathrm{dH})=0
$$

Therefore

$$
i\left(X_{\mu}\right) \Omega_{\mu}-\mathrm{dH}_{\mu}=j_{\mu}^{*}(i(X) \Omega-\mathrm{dH})=0
$$

and the second result follows.

In addition we have that: 
Lemma 1 ker $\Omega \subset \underline{\mathcal{X}\left(\mathcal{J}^{-1}(\mu)\right)}$. (That is, ker $\Omega$ lets $\mathcal{J}^{-1}(\mu)$ invariant).

( Proof) In fact, take the constraint functions $\{\zeta\}$ defining $\mathcal{J}^{-1}(\mu)$ such that $\mathrm{d} \zeta=i(\tilde{\xi}) \Omega$, for some $\tilde{\xi} \in \tilde{\mathbf{g}}$. Then, if $Z \in \operatorname{ker} \Omega$, we have that

$$
\mathrm{L}(Z) \zeta=i(Z) \mathrm{d} \zeta=i(Z) i(\tilde{\xi}) \Omega=-i(\tilde{\xi}) i(Z) \Omega=0
$$

therefore $Z \in \underline{\mathcal{X}\left(\mathcal{J}^{-1}(\mu)\right)}$.

Lemma $2 \tilde{\mathrm{g}}_{\mu_{x}}=\operatorname{ker} \Omega_{\mu_{x}} ;$ for every $x \in \mathcal{J}^{-1}(\mu)$.

( Proof) By proposition 1 we have that $\operatorname{ker} \Omega_{\mu_{x}}=\tilde{\mathbf{g}}_{\mu_{x}}+$ ker $\Omega_{x}$, and the assumption 2 gives us that ker $\Omega_{x} \subset \tilde{\mathbf{g}}_{x}$. On the other hand, $\tilde{\mathbf{g}}_{\mu_{x}}$ is the maximal subspace of $\tilde{\mathbf{g}}_{x}$ being tangent to $\mathcal{J}^{-1}(\mu)$; and ker $\Omega_{x}$ is made of vectors which are tangent to $\mathcal{J}^{-1}(\mu)$ (as a consequence of the above lemma). Therefore ker $\Omega_{x} \subset \tilde{\mathbf{g}}_{\mu_{x}}$, and the result follows.

Now, the last step is to obtain the orbit space $\left(\mathcal{J}^{-1}(\mu) / G_{\mu}, \hat{\Omega}\right)$.

Theorem 4 Consider the presymplectic Hamiltonian system $\left(\mathcal{J}^{-1}(\mu), \Omega_{\mu}, \mathrm{H}_{\mu}\right)$, the quotient manifold $\mathcal{J}^{-1}(\mu) / \operatorname{ker} \Omega_{\mu}$, and the canonical projection $\pi_{\mu}: \mathcal{J}^{-1}(\mu) \rightarrow \mathcal{J}^{-1}(\mu) /$ ker $\Omega_{\mu}$. Then the function $\mathrm{H}_{\mu}$ and the vector field $X_{\mu} \in \mathcal{X}\left(\mathcal{J}^{-1}(\mu)\right)$ of the proposition $\square$ are $\pi_{\mu}$-projectable. Hence $\left(\mathcal{J}^{-1}(\mu) / \operatorname{ker} \Omega_{\mu}, \hat{\Omega}, \hat{\mathrm{H}}\right)$, is a symplectic Hamiltonian system and

$$
i(\hat{X}) \hat{\Omega}-\mathrm{d} \hat{\mathrm{H}}=0
$$

where $\pi_{\mu}^{*} \hat{\mathrm{H}}=\mathrm{H}_{\mu}$ and $\pi_{\mu *} X_{\mu}=\hat{X}$.

( Proof ) According to the last lemma, we have that $\tilde{\mathbf{g}}_{\mu_{x}}=$ ker $\Omega_{\mu_{x}}$, for every $x \in \mathcal{J}^{-1}(\mu)$, and so $\mathcal{J}^{-1}(\mu) / G_{\mu}=\mathcal{J}^{-1}(\mu) / \operatorname{ker} \Omega_{\mu}$. Then, taking into account the first item of theorem 2 we have that $\left(\mathcal{J}^{-1}(\mu) / \operatorname{ker} \Omega_{\mu}, \hat{\Omega}\right)$ is a symplectic manifold.

Now, in order to see that $\mathrm{H}_{\mu}$ is $\pi_{\mu}$-projectable it suffices to prove that $\mathrm{L}\left(\tilde{\xi}_{\mu}\right) \mathrm{H}_{\mu}=0$, for every $\tilde{\xi}_{\mu} \in \tilde{\mathbf{g}}_{\mu} \subset \tilde{\mathbf{g}}$. But this holds since $\mathrm{H}$ is $G$-invariant and then $\mathrm{H}_{\mu}$ is $G_{\mu}$-invariant.

On the other hand, for every $\tilde{\xi}_{\mu} \in \tilde{\mathbf{g}}_{\mu}$, we have that

$$
i\left(\left[\tilde{\xi}_{\mu}, X_{\mu}\right]\right) \Omega_{\mu}=\mathrm{L}\left(\tilde{\xi}_{\mu}\right) i\left(X_{\mu}\right) \Omega_{\mu}-i\left(X_{\mu}\right) \mathrm{L}\left(\tilde{\xi}_{\mu}\right) \Omega_{\mu}=\mathrm{L}\left(\tilde{\xi}_{\mu}\right) \mathrm{dH}_{\mu}=0
$$

since $\Omega_{\mu}$ and $\mathrm{H}_{\mu}$ are $G_{\mu}$-invariant, and then $\left[\tilde{\xi}_{\mu}, X_{\mu}\right] \in \operatorname{ker} \Omega_{\mu}$. But, as all the elements of ker $\Omega_{\mu}$ are of the form $Z_{\mu}=f^{i} \xi_{\mu i}$, then we also have that $\left[Z_{\mu}, X_{\mu}\right] \in \operatorname{ker} \Omega_{\mu}$, for every $Z_{\mu} \in \operatorname{ker} \Omega_{\mu}$; and therefore, $X_{\mu}$ is $\pi_{\mu}$-projectable.

Finally, the equation (11) follows immediately from (10).

We can summarize the procedure in the following diagram

$$
(M, \Omega, \mathrm{H}) \stackrel{j_{\mu}}{\longleftarrow}\left(\mathcal{J}^{-1}(\mu), \Omega_{\mu}, \mathrm{H}_{\mu}\right) \stackrel{\pi_{\mu}}{\longrightarrow}\left(\mathcal{J}^{-1}(\mu) / \operatorname{ker} \Omega_{\mu}, \hat{\Omega}, \hat{\mathrm{H}}\right)
$$

The final result is a reduced symplectic dynamical system $\left(\mathcal{J}^{-1}(\mu) / \operatorname{ker} \Omega_{\mu}, \hat{\Omega}, \hat{\mathrm{H}}\right)$. Observe that, making only one quotient, we have removed the symmetries of the action of $G$ and the nonuniqueness arising from the existence of ker $\Omega$; then obtaining a symplectic dynamical system where $G$ acts by the identity.

From now on we will refer to this reduction scheme as the complete presymplectic reduction procedure. 


\subsection{Other reduction procedures: Gauge reduction plus symplectic reduction}

We finish this study by comparing the reduction method of presymplectic dynamical systems with symmetry here presented with the other step-by-step reduction procedures. In particular, the reduction procedure that has been developed in the last section, removes both rigid and gauge symmetries. Now, in this section, we make these procedures successively, proving that we obtain the same result as above.

Consider a compatible presymplectic dynamical system $(M, \Omega, \mathrm{H})$. Let $G$ be a group of symmetries of the presymplectic dynamical system, $\mathbf{g}$ its Lie algebra and $\tilde{\mathbf{g}}$ the corresponding algebra of fundamental vector fields. We suppose that the assumption 2 holds.

First, we apply the gauge reduction procedure obtaining the reduced phase space $(\bar{M}, \bar{\omega}, \overline{\mathrm{H}})$ (which is a symplectic dynamical system), with $\pi_{M}: M \rightarrow \bar{M}$. So the gauge symmetries have been removed. Next we must study under what conditions the action of $G$ goes down to $\bar{M}$ and, therefore, the corresponding non-gauge symmetry can be removed by means of the standard symplectic reduction procedure of Marsden-Weinstein.

Proposition 7 If $\Phi: G \times M \rightarrow M$ is a (strongly) presymplectic action, then there exists a "reduced action" $\bar{\Phi}: G \times \bar{M} \rightarrow \bar{M}$ such that $\bar{\Phi}_{g}\left(\pi_{M}(p)\right):=\pi_{M}\left(\Phi_{g}(p)\right)$; for every $g \in G$ and $p \in M$.

( Proof) In order to see that this reduced action $\bar{\Phi}$ is well defined, we must prove that, given $p_{1}, p_{2} \in M$ belonging to the same leaf of the foliation defined by ker $\Omega$, then $\Phi_{g}\left(p_{1}\right)=\Phi_{g}\left(p_{2}\right)$, for every $g \in G$. If $p_{1}, p_{2}$ are in the same leaf of this foliation, then they are connected by a (piecewise) regular curve, which is made of (pieces of) integral curves of vector fields belonging to ker $\Omega$. But, if $\gamma(t)$ is an integral curve of some $Z \in \operatorname{ker} \Omega$, then $\left(\Phi_{g} \circ \gamma\right)(t)$ is an integral curve of $\Phi_{g_{*}} Z$, which is also a vector field in ker $\Omega$ since, as $\Phi$ is a presymplectic action, we have

$$
i\left(\Phi_{g_{*}} Z\right) \Omega=i\left(\Phi_{g_{*}} Z\right)\left(\Phi_{g}^{-1}\right)^{*} \Omega=\left(\Phi_{g}^{-1}\right)^{*}(i(Z) \Omega)=0
$$

Therefore the action $\bar{\Phi}$ can be defined as is set in the statement.

Now the problem is that, although the action $\Phi$ can be assumed to be free, the reduced action $\bar{\Phi}$ is not so in general; because the assumption 2 implies that the leaves of the foliation induced by ker $\Omega$ are in the orbits of $G$ and, then, the quotient by ker $\Omega$ leads to a non free action, in general. Hence we set the following hypothesis (which is implicitly assumed in the physical literature):

Assumption 3 Let $\tilde{\mathbf{g}}$ be the vector space of the fundamental vector fields of the action $\Phi$ of the symmetry group $G$ on $M$. Then there is a subalgebra $\mathbf{G} \subset \mathbf{g}$, such that the corresponding $\tilde{\mathbf{G}} \subset \tilde{\mathbf{g}}$ verifies that $\operatorname{ker} \Omega \subset \mathrm{C}^{\infty}(M) \otimes \tilde{\mathbf{G}}$.

Now, suppose that $\mathcal{G} \subset G$ (the subgroup having $\mathbf{G}$ as Lie algebra) is closed; and let $\bar{G}:=G / \mathcal{G}$ be the quotient group, which acts on $\bar{M}$ by the reduced action $\bar{\Phi}$. Denote by $\mathbf{g}_{\bar{M}}$ the Lie algebra of $\bar{G}$, and by $\tilde{\mathbf{g}}_{\bar{M}}$ the corresponding set of fundamental vector fields. Then we have the projections

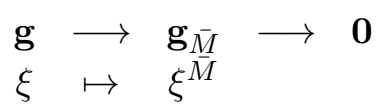

and the duals

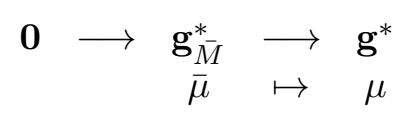

We have: 
Lemma 3 The set of fundamental vector fields $\tilde{\mathbf{g}}$ is $\pi_{M}$-projectable.

( Proof) In fact, for every $\tilde{\xi} \in \tilde{\mathbf{g}}$ and for every $Z \in$ ker $\Omega$, we have that

$$
i([\tilde{\xi}, Z]) \Omega=\mathrm{L}(\tilde{\xi}) i(Z) \Omega-i(Z) \mathrm{L}(\tilde{\xi}) \Omega=0
$$

therefore $[\tilde{\xi}, Z] \in \operatorname{ker} \Omega$ and the result follows.

And therefore:

Proposition 8 With the above assumptions, if the action $\Phi$ of $G$ on $M$ is strongly presymplectic and free, then the reduced action $\bar{\Phi}$ of the quotient group $\bar{G}$ on $\bar{M}$ is strongly symplectic and free.

( Proof) First we prove that $\bar{\Phi}$ is free. In fact; if $\bar{g} \in \bar{G}$ and $p \in M$, then $\bar{\Phi}_{\bar{g}}\left(\pi_{M}(p)\right)=\pi_{M}(p)$, by definition. But, if $\bar{\Phi}_{g}\left(\pi_{M}(p)\right)=\pi_{M}(p)$, for some $g \in G$, then $\pi_{M}\left(\Phi_{g}(p)=\pi_{M}(p)\right.$, and hence $g \in \mathcal{G}$. Therefore the isotropy group of a point of the action of $G$ on $M$ is $\mathcal{G}$, thus the action of $\bar{G}$ on $\bar{M}$ is free.

Second we prove that $\bar{\Phi}$ is strongly symplectic. In fact; for every $\tilde{\xi} \in \tilde{\mathbf{g}}$ with $i(\tilde{\xi}) \Omega=\mathrm{d} f_{\xi}$, we have that $f_{\xi}$ is $\pi_{M}$-projectable. In fact

$$
\mathrm{L}(Z) f_{\xi}=i(Z) \mathrm{d} f_{\xi}=i(Z) i(\tilde{\xi}) \Omega=-i(\tilde{\xi}) i(Z) \Omega=0
$$

for every $Z \in$ ker $\Omega$. Then the function $\bar{f}_{\xi} \in \mathrm{C}^{\infty}(\bar{M})$ such that $\pi_{M}^{*} \bar{f}_{\xi}=f_{\xi}$ is Hamiltonian for the fundamental vector field $\tilde{\xi}^{\bar{M}} \in \tilde{\mathbf{g}}_{\bar{M}}$ such that $\pi_{M_{*}} \tilde{\xi}=\tilde{\xi}^{\bar{M}}$, since

$$
\pi_{M}^{*} \mathrm{~d} \bar{f}_{\xi}=\mathrm{d} \pi_{M}^{*} \bar{f}_{\xi}=\mathrm{d} f_{\xi}=i(\tilde{\xi}) \Omega=i(\tilde{\xi}) \pi_{M}^{*} \bar{\omega}=\pi_{M}^{*} i\left(\tilde{\xi}^{\bar{M}}\right) \bar{\omega}
$$

and hence $\mathrm{d} \bar{f}_{\xi}=i\left(\tilde{\xi}^{\bar{M}}\right) \bar{\omega}$, since $\pi_{M}$ is a submersion.

Now, we can define the reduced comomentum mapping associated with $\bar{\Phi}$ as a Lie algebra linear map

$$
\begin{aligned}
& \overline{\mathcal{J}}^{*}: \mathbf{g}_{\bar{M}} \rightarrow \mathrm{C}^{\infty}(\bar{M}) \\
& \xi^{\bar{M}} \mapsto \quad \bar{f}_{\xi}
\end{aligned}
$$

such that $i\left(\tilde{\xi}^{\bar{M}}\right) \bar{\omega}=\mathrm{d} \bar{f}_{\xi}$. The reduced momentum mapping associated with $\bar{\Phi}$ is its dual map, $\overline{\mathcal{J}}: \bar{M} \rightarrow \mathbf{g}_{\bar{M}}^{*}$; that is, for every $\xi^{\bar{M}} \in \mathbf{g}_{\bar{M}}$ and $x \in M$,

$$
\left(\overline{\mathcal{J}}\left(\pi_{M}(x)\right)\right)\left(\xi^{\bar{M}}\right):=\overline{\mathcal{J}}^{*}\left(\xi^{\bar{M}}\right)\left(\pi_{M}(x)\right)=\bar{f}_{\xi}\left(\pi_{M}(x)\right)
$$

Finally, it can be proved that if $\Phi$ is a Poissonian and proper action, then so is $\bar{\Phi}$.

At this point, the standard symplectic reduction program is applied:

- First we construct the level sets $\left(\overline{\mathcal{J}}^{-1}(\bar{\mu}), \omega_{\bar{\mu}}, \mathrm{H}_{\bar{\mu}}\right)$, for weakly regular values $\bar{\mu} \in \mathbf{g}_{\bar{M}}^{*}$. Each one of them is locally defined in $(\bar{M}, \bar{\omega})$ by the constraints $\left\{\bar{f}_{\xi}\right\}$.

- Second we take the isotropy group $\bar{G}_{\bar{\mu}}$ and its Lie algebra $\left(\mathbf{g}_{\bar{M}}\right)_{\bar{\mu}}$, for which we have that $\left(\tilde{\mathbf{g}}_{\bar{M}}\right)_{\bar{\mu}}=\operatorname{ker} \omega_{\bar{\mu}}$. Then we make the quotient $\overline{\mathcal{J}}^{-1}(\bar{\mu}) / \bar{G}_{\bar{\mu}}$.

After that, the reduced symplectic Hamiltonian system $\left(\overline{\mathcal{J}}^{-1}(\bar{\mu}) / \operatorname{ker} \omega_{\bar{\mu}}, \hat{\omega}, \hat{\mathrm{h}}\right)$ is free of gauge and rigid symmetries and the following theorem proves that it coincides with $\left(\mathcal{J}^{-1}(\mu) / \operatorname{ker} \Omega_{\mu}, \hat{\Omega}, \hat{\mathrm{H}}\right)$, which is the one obtained after the complete presymplectic reduction procedure (for $\mu$ and $\bar{\mu}$ related as shown in (12)). 
Theorem 5 There exists a diffeomorphism

$$
\rho:\left(\mathcal{J}^{-1}(\mu) / \operatorname{ker} \Omega_{\mu}, \hat{\Omega}, \hat{\mathrm{H}}\right) \longrightarrow\left(\overline{\mathcal{J}}^{-1}(\bar{\mu}) / \operatorname{ker} \omega_{\bar{\mu}}, \hat{\omega}, \hat{\mathrm{h}}\right)
$$

such that $\hat{\Omega}=\rho^{*} \hat{\omega}$ and $\hat{\mathrm{H}}=\rho^{*} \hat{\mathrm{h}}$.

( Proof) First of all, we can construct a map $\tau:\left(\mathcal{J}^{-1}(\mu), \Omega_{\mu}, \mathrm{H}_{\mu}\right) \rightarrow\left(\overline{\mathcal{J}}^{-1}(\bar{\mu}), \omega_{\bar{\mu}}, \mathrm{H}_{\bar{\mu}}\right)$ verifying the relation $j_{\bar{\mu}} \circ \tau=\pi_{M} \circ j_{\mu}$ (see the diagram below). Observe that, if $p \in \mathcal{J}^{-1}(\mu)$ then $\pi_{M}(p) \in \overline{\mathcal{J}}^{-1}(\bar{\mu})$, by the proposition 8 and the relation between $\mu$ and $\bar{\mu}$ (see (12)); hence $\tau$ is well defined and it is a surjective submersion (since so is $\pi_{M}$ ). Moreover, we have that

$$
\Omega_{\mu}=j_{\mu}^{*} \pi_{M}^{*} \bar{\omega}=\tau^{*} j_{\bar{\mu}}^{*} \bar{\omega}=\tau^{*} \omega_{\bar{\mu}}
$$

and in the same way we obtain that $\mathrm{H}_{\mu}=\tau^{*} \mathrm{H}_{\bar{\mu}}$.

Now there is an unique map $\rho:\left(\mathcal{J}^{-1}(\mu) / \operatorname{ker} \Omega_{\mu}, \hat{\Omega}, \hat{\mathrm{H}}\right) \longrightarrow\left(\overline{\mathcal{J}}^{-1}(\bar{\mu}) / \operatorname{ker} \omega_{\bar{\mu}}, \hat{\omega}, \hat{\mathrm{h}}\right)$ such that $\rho \circ \pi_{\mu}=\tau \circ \pi_{\bar{\mu}}$. So we have the diagram

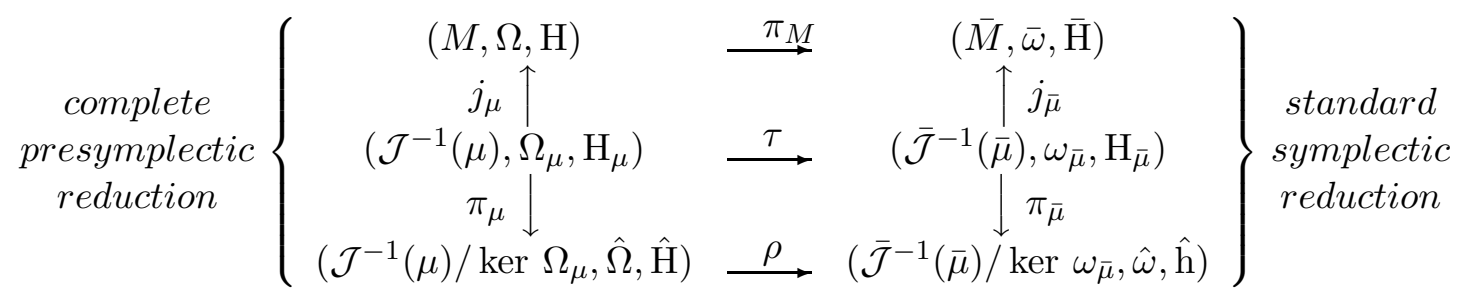

The map $\rho$ has the following properties:

1. $\rho$ is well defined:

Let $p_{1}, p_{2} \in \mathcal{J}^{-1}(\mu)$ such that $\pi_{\mu}\left(p_{1}\right)=\pi_{\mu}\left(p_{2}\right)$; we have to prove that $\pi_{\bar{\mu}} \tau\left(p_{1}\right)=\pi_{\bar{\mu}} \tau\left(p_{2}\right)$. Since $\pi_{\mu}\left(p_{1}\right)=\pi_{\mu}\left(p_{2}\right)$, it implies that $p_{1}, p_{2}$ can be joined by a polygonal made of integral curves of vector fields of ker $\Omega_{\mu}$ (but we will take a single curve, since it suffices to repeat the reasoning a finite number of times). For every point $p$ of the curve, if $Z_{p} \in \mathrm{T}_{p} \mathcal{J}^{-1} \mu$ is tangent to this curve at $p$, as $\tau$ is a surjective submersion and $\tau^{*} \omega_{\bar{\mu}}=\Omega_{\mu}$, we have that

$$
i\left(Z_{p}\right)\left(\Omega_{\mu}\right)_{p}=i\left(Z_{p}\right)\left(\tau^{*} \omega_{\bar{\mu}}\right)_{p}=\tau_{p}^{*} i\left(\mathrm{~T}_{p} \tau\left(Z_{p}\right)\right)\left(\omega_{\bar{\mu}}\right)_{\pi_{\tau(p)}}=0
$$

As a consequence $\left.\mathrm{T}_{p} \tau\right|_{\operatorname{ker}\left(\Omega_{\mu}\right)_{p}}: \operatorname{ker}\left(\Omega_{\mu}\right)_{p} \rightarrow \operatorname{ker}\left(\omega_{\bar{\mu}}\right)_{\tau(p)}$ is surjective and ker $\mathrm{T}_{p} \tau \subset \operatorname{ker}\left(\Omega_{\mu}\right)_{p}$. Hence, the curve joining $p_{1}$ and $p_{2}$ can be covered by a finite number of open sets satisfying this property and, then, $\tau\left(p_{1}\right)$ and $\tau\left(p_{2}\right)$ are connected by a polygonal of integral curves of vector fields of ker $\omega_{\bar{\mu}}$; that is, $\pi_{\bar{\mu}} \tau\left(p_{1}\right)=\pi_{\bar{\mu}} \tau\left(p_{2}\right)$.

2. $\rho$ is bijective:

$\tau$ maps the leaves of the foliation defined by ker $\Omega_{\mu}$ into the leaves of the foliation defined by ker $\omega_{\bar{\mu}}$; therefore $\rho$ is injective. To see that $\rho$ is surjective is trivial.

3. $\rho$ is a diffeomorphism:

$\pi_{\mu}: \mathcal{J}^{-1}(\mu) \rightarrow \mathcal{J}^{-1}(\mu) /$ ker $\Omega_{\mu}$ is a surjective submersion, then there are differentiable local sections $s_{\mu}: \mathcal{J}^{-1}(\mu) / \operatorname{ker} \Omega_{\mu} \rightarrow \mathcal{J}^{-1}(\mu)$ (that is, such that $\pi_{\mu} \circ s_{\mu}=\mathrm{Id}$ ); hence, locally, we have that $\rho=\pi_{\bar{\mu}} \circ \tau \circ s_{\mu}$ and then $\rho$ is differentiable since so are $\pi_{\bar{\mu}}, \tau$ and $s_{\mu}$. (The choice of the local sections $s_{\mu}$ is called gauge fixing in the physical literature).

Now we must prove that $\rho^{-1}$ is differentiable. Taking into account the theorem of the inverse function, it is sufficient to prove that the tangent map $\mathrm{T}_{\pi_{\mu}(p)} \rho$ is an isomorphism 
for every $p \in \mathcal{J}^{-1}(\mu)$. Then, let $u \in \mathrm{T}_{\pi_{\mu}(p)}\left(\mathcal{J}^{-1}(\mu) / \operatorname{ker} \Omega_{\mu}\right)$ such that $\mathrm{T}_{\pi_{\mu}(p)} \rho(u)=0$ and $v \in\left(\mathrm{T}_{p} \pi_{\mu}\right)^{-1}(u)$. By the commutativity of the diagram above we have that $\mathrm{T}_{p}\left(\pi_{\bar{\mu}} \circ\right.$ $\tau)(v)=0$; hence $\mathrm{T}_{p} \tau(v) \in \operatorname{ker} \mathrm{T}_{\tau(p)} \pi_{\bar{\mu}}=\operatorname{ker}\left(\omega_{\bar{\mu}}\right)_{\tau(p)}$ and therefore $v \in \operatorname{ker}\left(\Omega_{\mu}\right)_{p}$ because $\left(\mathrm{T}_{p} \tau\right)^{-1}\left(\operatorname{ker}\left(\omega_{\bar{\mu}}\right)_{\tau(p)}\right)=\operatorname{ker}\left(\Omega_{\mu}\right)_{p}$. But $\operatorname{ker}\left(\Omega_{\mu}\right)_{p}=\operatorname{ker} \mathrm{T}_{p} \pi_{\mu}$, therefore $u=\mathrm{T}_{p} \pi_{\mu}(v)=0$, hence $\mathrm{T}_{\pi_{\mu}(p)} \rho$ is injective and, as a consequence, it is an isomorphism.

4. $\rho$ is a symplectomorphism:

On the one hand $\Omega_{\mu}=\pi_{\mu}^{*} \hat{\Omega}$, but on the other hand

$$
\Omega_{\mu}=\tau^{*} \omega_{\bar{\mu}}=\tau^{*} \pi_{\bar{\mu}}^{*} \hat{\omega}=\pi_{\mu}^{*} \rho^{*} \hat{\omega}
$$

hence $\pi_{\mu}^{*} \hat{\Omega}=\pi_{\mu}^{*} \rho^{*} \hat{\omega}$ and, since $\pi_{\mu}$ is a submersion, we have that $\hat{\Omega}=\rho^{*} \hat{\omega}$.

5. The proof for $\mathrm{H}_{\mu}=\tau^{*} \mathrm{H}_{\bar{\mu}}$ is like in the last item.

\subsection{Other reduction procedures: Coisotropic imbedding plus symplectic reduc- tion}

Let $(M, \Omega, \mathrm{H})$ be a compatible presymplectic dynamical system and $(\mathbf{M}, \boldsymbol{\Omega}, \mathbf{H})$ an ambient symplectic system associated to it (see section 3.1). If the presymplectic dynamical system exhibits non-gauge symmetries as well as gauge symmetries, under certain hypothesis, both can be removed applying the standard symplectic reduction procedure of Marsden-Weinstein to the symplectic dynamical system $(\mathbf{M}, \boldsymbol{\Omega}, \mathbf{H})$. On the other hand, we can apply the presymplectic reduction method here explained and then we will prove that both procedures also lead to the same final result.

Let $G$ be a group of symmetries of the presymplectic dynamical system, $\mathbf{g}$ its Lie algebra and $\tilde{\mathbf{g}} \subset \mathcal{X}_{h}(M)$ the corresponding set of fundamental vector fields. First of all, using the coisotropic imbedding theorem, it can be proved [14] that, for every presymplectomorphism $\Phi_{g}:(M, \Omega) \rightarrow$ $(M, \Omega)$, there exists a symplectomorphism $\mathbf{\Phi}_{g}:(\mathbf{M}, \boldsymbol{\Omega}) \rightarrow(\mathbf{M}, \boldsymbol{\Omega})$ such that it reduces to $\Phi_{g}$ on $M$; that is, $\boldsymbol{\Phi}_{g} \circ j_{0}=j_{0} \circ \Phi_{g}$. Taking this into account, we will assume that:

Assumption 4 The presymplectic action $\Phi: M \times G \rightarrow M$ can be extended to a symplectic action $\mathbf{\Phi}: \mathbf{M} \times G \rightarrow \mathbf{M}$ which reduces to $\Phi$ on $M \times G$, that is, $\boldsymbol{\Phi}_{g} \circ\left(j_{0} \times \operatorname{Id}_{G}\right)=j_{0} \circ \Phi_{g}$ (and it is also assumed to be Poissonian, free and proper).

Then, denoting by $\tilde{\mathbf{g}}_{\mathbf{M}} \subset \mathcal{X}_{h}(\mathbf{M})$ the set of fundamental vector fields for this extended action, it is obvious that $\tilde{\mathbf{g}}_{\mathbf{M}} \subset \underline{\mathcal{X}(M)}$ and then, for every $\tilde{\xi}^{\prime} \in \tilde{\mathbf{g}}_{\mathbf{M}}$, there exists one $\tilde{\xi} \in \tilde{\mathbf{g}}$ such that $j_{0 *} \tilde{\xi}=\left.\tilde{\xi}^{\prime}\right|_{M}$, and conversely.

Let $\mathbf{J}: \mathbf{M} \rightarrow \mathbf{g}^{*}$ be a momentum map associated with the extended action $\mathbf{\Phi}$. Once again, the standard symplectic reduction program can be applied:

- First constructing the level sets $\left(\mathbf{J}^{-1}(\mu), \boldsymbol{\Omega}_{\mu}, \mathbf{H}_{\mu}\right)$, for weakly regular values $\mu \in \mathbf{g}^{*}$ of $\mathbf{J}$. Each one is locally defined in $(\mathbf{M}, \boldsymbol{\Omega})$ by the constraints $\left\{\mathbf{f}_{\xi}\right\}$ which are the Hamiltonian functions of the vector fields of $\tilde{\mathbf{g}}_{\mathbf{M}}$.

- Second taking the isotropy group $G_{\mu}$ and its Lie algebra $\mathbf{g}_{\mu}$, for which we have $\tilde{\mathbf{g}}_{\mu}=\operatorname{ker} \boldsymbol{\Omega}_{\mu}$, and constructing the quotient $\mathbf{J}^{-1}(\mu) / G_{\mu}$. 
After that, the corresponding reduced symplectic Hamiltonian system $\left(\mathbf{J}^{-1}(\mu) /\right.$ ker $\left.\boldsymbol{\Omega}_{\mu}, \hat{\boldsymbol{\Omega}}, \hat{\mathbf{H}}\right)$ is free of symmetries.

On the other hand, we can consider the momentum map $\mathcal{J}: M \rightarrow \mathbf{g}^{*}$ which is compatible with $\mathbf{J}$. This means that $\mathcal{J}$ is induced by $\mathbf{J}$ on $M$; that is, $\mathcal{J}:=\mathbf{J} \circ j_{0}$. We can then apply the complete presymplectic reduction procedure constructing the level sets $\left(\mathcal{J}^{-1}(\mu), \Omega_{\mu}, \mathrm{H}_{\mu}\right)$, for weakly regular values $\mu \in \mathbf{g}^{*}$ of $\mathcal{J}$, and the quotient $\mathcal{J}^{-1}(\mu) / G_{\mu}$. So we have the (commutative) diagram

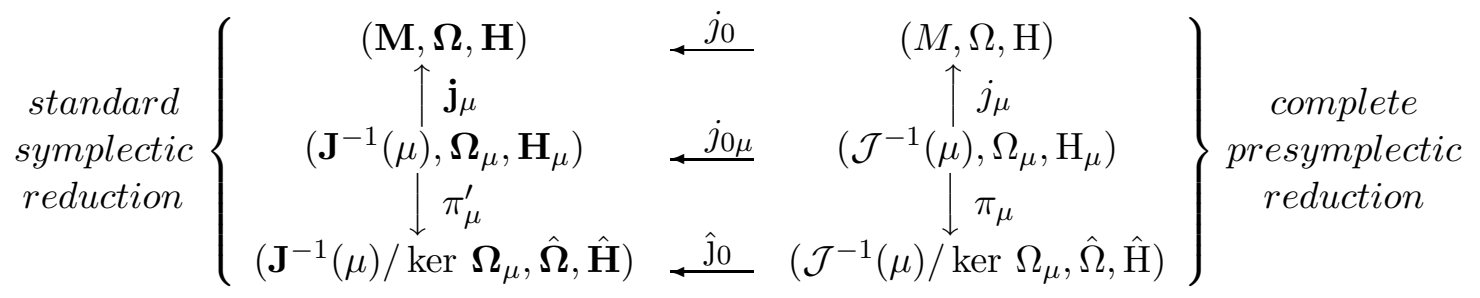

Then, if $\mu \in \mathbf{g}^{*}$ is a weakly regular value for $\mathbf{J}$ and $\mathcal{J}$, we wish to compare the sets $\left(\mathbf{J}^{-1}(\mu), \boldsymbol{\Omega}_{\mu}, \mathbf{H}_{\mu}\right)$ and $\left(\mathbf{J}^{-1}(\mu) / \operatorname{ker} \boldsymbol{\Omega}_{\mu}, \hat{\boldsymbol{\Omega}}, \hat{\mathbf{H}}\right)$ with $\left(\mathcal{J}^{-1}(\mu), \Omega_{\mu}, \mathrm{H}_{\mu}\right)$ and $\left(\mathcal{J}^{-1}(\mu) / \operatorname{ker} \Omega_{\mu}, \hat{\Omega}, \hat{\mathrm{H}}\right)$ respectively. So we have:

Theorem 6 With the conditions stated in the assumption 母:

1. $j_{0 \mu}\left(\mathcal{J}^{-1}(\mu)\right)$ is a connected component of $\mathbf{J}^{-1}(\mu)$, or a union of connected components of it. Moreover, $j_{0 \mu}^{*} \boldsymbol{\Omega}_{\mu}=\Omega_{\mu}$ and $j_{0 \mu}^{*} \mathbf{H}_{\mu}=\mathrm{H}_{\mu}$.

2. $\hat{\mathrm{j}}_{0}\left(\mathcal{J}^{-1}(\mu) / \operatorname{ker} \Omega_{\mu}\right)$ is a connected component of the quotient $\mathbf{J}^{-1}(\mu) / \operatorname{ker} \boldsymbol{\Omega}_{\mu}$, or a union of connected components of it. Moreover, $\hat{\mathrm{j}}_{0}^{*} \hat{\boldsymbol{\Omega}}=\hat{\Omega}$ and $\hat{\mathrm{j}}_{0}^{*} \hat{\mathbf{H}}=\hat{\mathrm{H}}$.

(Proof) Let $\left\{\xi_{i}\right\}$ be a basis of $\mathbf{g}$ and $\mathbf{f}_{\xi_{i}} \in \mathrm{C}^{\infty}(\mathbf{M})$ Hamiltonian functions for the corresponding $\tilde{\xi}_{i}^{\prime} \in \tilde{\mathbf{g}}_{\mathbf{M}}$. Let $\left\{\alpha^{i}\right\}$ be the dual basis in $\mathbf{g}^{*}$ and $\mu=\mu_{i} \alpha^{i}$ (with $\mu_{i} \in \mathbf{R}$ ) a weakly regular value for $\mathbf{J}$ and $\mathcal{J}$.

First, we are going to prove that, f the submanifold $\mathbf{J}^{-1}(\mu) \hookrightarrow \mathbf{M}$ is locally defined by the constraints $\zeta_{i}:=\mathbf{f}_{\xi_{i}}-\mu_{i}$, then the constraints $j_{0}^{*} \zeta_{i}$ define locally the submanifold $\mathcal{J}^{-1}(\mu) \hookrightarrow \mathbf{M}$. In fact; as $\mathcal{J}:=\mathbf{J} \circ j_{0}$ we have that $\mathbf{J}(x)=\mathcal{J}(x)$, for every $x \in M$, and, taking into account (5), this implies that $j_{0}^{*} \mathbf{f}_{\xi_{i}}(x) \alpha^{i}=f_{\xi_{i}}(x) \alpha^{i}$ and thus $j_{0}^{*} \mathbf{f}_{\xi_{i}}=f_{\xi_{i}}$. Therefore:

- If $\tilde{\xi}_{i}^{\prime} \notin \underline{\text { ker } \Omega}$ (that is, it generates infinitesimal non-gauge symmetries), then the corresponding $\tilde{\xi}_{i} \in \mathcal{X}(M)$ is not in ker $\Omega$ and hence

$$
\mathrm{d} j_{0}^{*} \mathbf{f}_{\xi_{i}}=j_{0}^{*} \mathrm{~d} \mathbf{f}_{\xi_{i}}=j_{0}^{*}\left(i\left(\tilde{\xi}_{i}^{\prime}\right) \boldsymbol{\Omega}\right)=i\left(\tilde{\xi}_{i}\right) \Omega=\mathrm{d} f_{\xi_{i}}
$$

As $\mathrm{d} f_{\xi_{i}} \neq 0$ then $j_{0}^{*} \mathbf{f}_{\xi_{i}}$ is not constant on $M$, but $j_{\mu}^{*} j_{0}^{*} \mathbf{f}_{\xi_{i}}$ is; hence $\mathbf{f}_{\xi_{i}}$ are constraints for $\mathcal{J}^{-1}(\mu) \hookrightarrow \mathbf{M}$ necessarily, but not for $M \hookrightarrow \mathbf{M}$.

Conversely, every constraint function $\zeta \in \mathrm{C}^{\infty}(M)$ for $\mathcal{J}^{-1}(\mu) \hookrightarrow \mathbf{M}$ can be extended to a function of $\zeta^{\prime} \in \mathrm{C}^{\infty}(\mathbf{M})$ such that its Hamiltonian vector field does not belong to $\underline{\mathrm{ker} \Omega}$.

- If $\tilde{\xi}_{i}^{\prime} \in \underline{\operatorname{ker} \Omega}$ (that is, it generates infinitesimal gauge symmetries), then the corresponding $\tilde{\xi}_{i} \in \mathcal{X}(M)$ is in ker $\Omega$ and hence

$$
0=i\left(\tilde{\xi}_{i}\right) \Omega=i\left(\tilde{\xi}_{i}\right)\left(j_{0}^{*} \boldsymbol{\Omega}\right)=j_{0}^{*}\left(i\left(\tilde{\xi}_{i}^{\prime}\right) \boldsymbol{\Omega}\right)=j_{0}^{*} \mathrm{~d} \mathbf{f}_{\xi_{i}}=\mathrm{d} j_{0}^{*} \mathbf{f}_{\xi_{i}}
$$

So $\mathbf{f}_{\xi_{i}}$ is constant on $M$ and therefore it is a constraint for $\mathcal{J}^{-1}(\mu) \hookrightarrow \mathbf{M}$, as well as for $M \hookrightarrow \mathbf{M}$.

Conversely, for every constraint function $\phi$ for $M \hookrightarrow \mathbf{M}$, its Hamiltonian vector field necessarily belongs to $\underline{\operatorname{ker} \Omega}$. 
On the other hand, since $j_{0}$ is a coisotropic imbedding, we have that $\operatorname{dim}(\operatorname{ker} \Omega)=\operatorname{dim} \mathbf{M}-\operatorname{dim} M$; and as $\operatorname{dim}\left(\mathbf{J}^{-1}(\mu)\right)$ (in $\mathbf{M}$ ) is equal to $\operatorname{dim} \mathbf{M}-\operatorname{dim} \mathbf{g}$, we obtain that

$$
\operatorname{dim}\left(\mathcal{J}^{-1}(\mu)\right)=\operatorname{dim} \mathbf{M}-(\operatorname{dim} \mathbf{g}-\operatorname{dim}(\operatorname{ker} \Omega))=\operatorname{dim} M-\operatorname{dim} \mathbf{g}=\operatorname{dim}\left(\mathbf{J}^{-1}(\mu)\right)
$$

Hence we conclude that $j_{0 \mu}\left(\mathcal{J}^{-1}(\mu)\right)$ is a submanifold of $\mathbf{J}^{-1}(\mu)$ and, as both have the same dimension, we can conclude that $j_{0 \mu}\left(\mathcal{J}^{-1}(\mu)\right)$ is open in $\mathbf{J}^{-1}(\mu)$. So, it is a connected component of $\mathbf{J}^{-1}(\mu)$, or a union of connected components of it (remember that both manifolds are closed, since they are defined by constraints). In addition,

$$
j_{0 \mu}^{*} \boldsymbol{\Omega}_{\mu}=j_{0 \mu}^{*} \mathbf{j}_{\mu}^{*} \boldsymbol{\Omega}=j_{\mu}^{*} j_{0}^{*} \boldsymbol{\Omega}=\Omega_{\mu}
$$

and, in the same way $j_{0 \mu}^{*} \mathbf{H}_{\mu}=\mathrm{H}_{\mu}$.

Finally, the results for the reduced phase spaces follow immediately from here.

A particular case of this reduction procedure (coisotropic imbedding plus symplectic reduction) is when ker $\Omega=\mathrm{C}^{\infty}(M) \otimes \tilde{\mathbf{g}}_{M}$. Then $\left(\mathbf{J}^{-1}(\mu), \boldsymbol{\Omega}, \mathbf{H}\right)=(M, \Omega, \mathrm{H})$ and $\left(\mathbf{J}^{-1}(\mu) / \operatorname{ker} \boldsymbol{\Omega}, \hat{\boldsymbol{\Omega}}, \hat{\mathbf{H}}\right)=$ $(\bar{M}, \bar{\omega}, \overline{\mathrm{H}})$. In this case, this method is the generalized symplectic reduction studied in [29] and [37]. (See also 45 for a study on other features on this topic).

The successive steps of the three reduction procedures here analyzed can be summarized in the following diagram

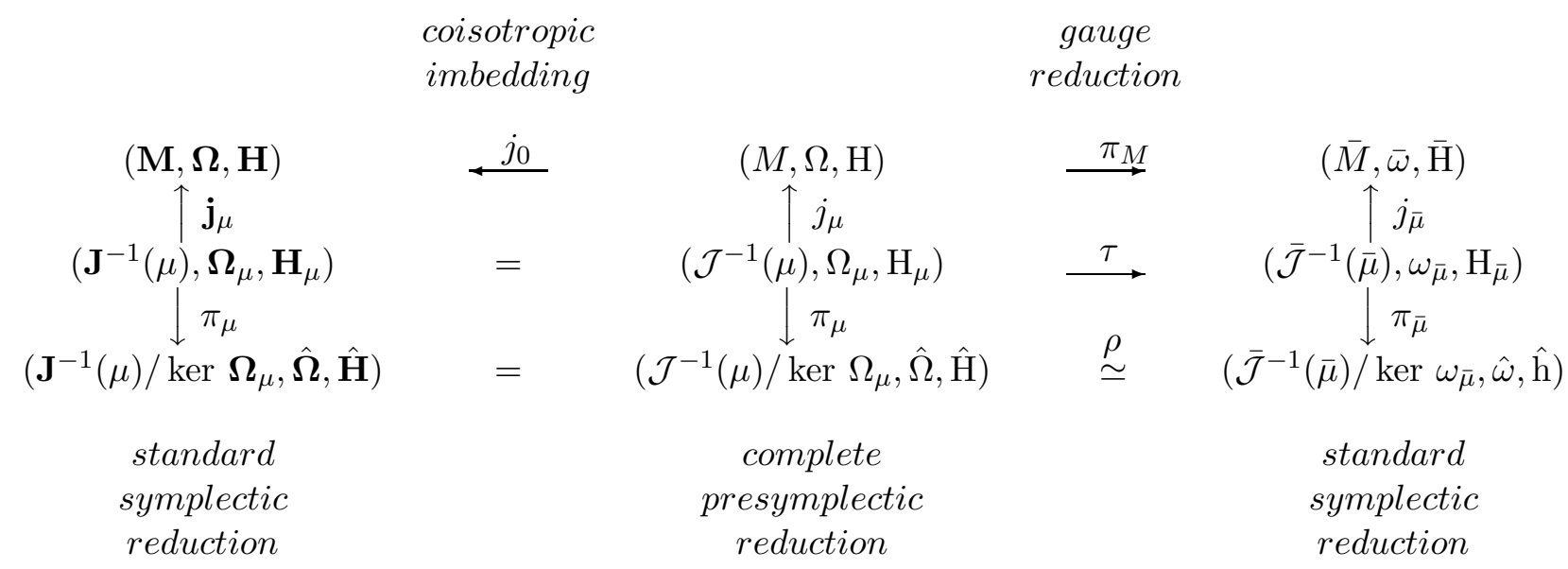

(where the equalities mean that the imbeddings of the manifolds in the center column are (a union of) connected components of the corresponding manifolds in the left column).

\subsection{Reduction of non-compatible presymplectic dynamical systems with sym- metry}

The concept of group of symmetries can also be established for a non-compatible presymplectic dynamical system $(P, \omega, \mathcal{H})$ with final compatible system $(M, \Omega, \mathrm{H})$ (where $M$ is the final constraint submanifold). Thus, from the definition 1 we state:

Definition 5 Let $(P, \omega, \mathcal{H})$ be a non-compatible presymplectic dynamical system with final compatible system $(M, \Omega, \mathrm{H}), G$ a Lie group and $\Psi: G \times P \rightarrow P$ an action of $G$ on $P$. $G$ is said to be a symmetry group of this system iff

1. $\Psi$ leaves $M$ invariant; that is, it induces an action $\Phi: G \times M \rightarrow M$ such that $\Psi \circ\left(\operatorname{Id}_{G} \times j_{M}\right)=$ $j_{M} \circ \Phi$. 
2. The induced action $\Phi$ is a presymplectic action on $(M, \Omega)$ (which is assumed to be Poissonian, free and proper); that is, for every $g \in G$,

$$
j_{M}^{*}\left(\Psi_{g}^{*} \omega-\omega\right)=\Phi_{g}^{*} \Omega-\Omega=0
$$

(Following a very usual terminology in physics, we will say that $\Psi$ is a weakly presymplectic action on $(P, \omega, M))$.

3. For every $g \in G$,

$$
j_{M}^{*}\left(\Psi_{g}^{*} \mathcal{H}-\mathcal{H}\right)=\Phi_{g}^{*} \mathrm{H}-\mathrm{H}=0
$$

Of course all the results discussed in the above sections hold for the compatible dynamical system $(M, \Omega, \mathrm{H})$ and the action $\Phi$. In particular, let $\mathbf{g}$ be the Lie algebra of $G$ and $\tilde{\mathbf{g}}_{M}$ and $\tilde{\mathbf{g}}_{P}$ the sets of fundamental vector fields on $M$ and $P$ (with respect to the actions $\Phi$ and $\Psi$ ) respectively. Then, for every $\xi \in \mathbf{g}$, there exist $\tilde{\xi}^{M} \in \tilde{\mathbf{g}}_{M}$ and $\tilde{\xi}^{P} \in \tilde{\mathbf{g}}_{P}$, with $j_{M_{*}} \tilde{\xi}^{M}=\left.\tilde{\xi}^{P}\right|_{M}$, such that

$$
\begin{aligned}
j_{M}^{*} \mathrm{~L}\left(\tilde{\xi}^{P}\right) \mathcal{H} & =\mathrm{L}\left(\tilde{\xi}^{M}\right) \mathrm{H}=0 \\
j_{M}^{*} \mathrm{~L}\left(\tilde{\xi}^{P}\right) \omega & =\mathrm{L}\left(\tilde{\xi}^{M}\right) \Omega=0
\end{aligned}
$$

As the level sets of the momentum map associated to the action $\Phi$ are submanifolds of $M$, $\mathcal{J}^{-1}(\mu) \hookrightarrow M$, we may ask how are they defined as submanifolds of $P$. We have the following diagram

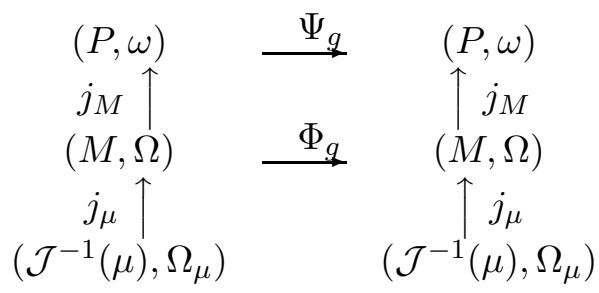

Taking into account the above discussion, we have that the constraint functions $\zeta_{i}^{M} \in \mathrm{C}^{\infty}(M)$ defining $\mathcal{J}^{-1}(\mu)$ in $M$ can be extended to $P$ as functions $\zeta_{i}^{P} \in \mathrm{C}^{\infty}(P)$ such that $j_{M}^{*} \zeta_{i}^{P}=\zeta_{i}^{M}$ since, if $\left\{\xi_{i}\right\}$ is a basis of $\mathbf{g}$, we have

$$
\mathrm{d} \zeta_{i}^{M}:=i\left(\tilde{\xi}_{i}^{M}\right) \Omega=j_{M}^{*}\left(i\left(\tilde{\xi}_{i}^{P}\right) \omega\right)=j_{M}^{*} \mathrm{~d} \zeta_{i}^{P}=\mathrm{d}\left(j_{M}^{*} \zeta_{i}^{P}\right)
$$

Hence the submanifolds $\mathcal{J}^{-1}(\mu) \hookrightarrow P$ are defined in $P$ by these constraints $\zeta^{P}$ together with the constraints $\eta$ defining $M$ in $P$.

As a particular case of special interest we have:

Proposition 9 With the conditions of definition 5, and assuming the following hypothesis:

1. Assumption 专 holds for $(M, \Omega)$.

2. There is a basis of constraint functions $\left\{\eta_{i}\right\}$ defining $M$ in $P$ made of presymplectic Hamiltonian functions in $(P, \omega)$.

3. The presymplectic Hamiltonian vector fields $X_{\eta_{i}} \in \mathcal{X}_{h}(P)$ associated to these constraints are tangent to $M$.

Then the momentum map $\mathcal{J}: M \rightarrow \mathbf{g}^{*}$ can be extended to a map $\mathrm{J}: P \rightarrow \mathbf{g}^{*}$ such that $\mathcal{J}=\mathrm{J} \circ j_{M}$ and $\mathcal{J}^{-1}(\mu)=\mathrm{J}^{-1}(\mu)$. 
(Proof) First of all, from items 2 and 3 we have that $X_{\eta_{i}}$ expand locally the set ker $\Omega$ 22], [7] and, from item 1, we have also that $X_{\eta_{i}} \in \tilde{\mathbf{g}}$. Then, the map $\mathrm{J}: P \rightarrow \mathbf{g}^{*}$ is defined in the following way: for every $\xi \in \mathrm{g}$ let $f_{\xi}^{M} \in \mathrm{C}^{\infty}(M)$ be the presymplectic Hamiltonian function of the fundamental vector field $\tilde{\xi}^{M} \in \mathcal{X}(M)$ and $f_{\xi}^{P} \in \mathrm{C}^{\infty}(P)$ its extension to $P$; then, for every $p \in P$,

$$
(\mathrm{J}(p))(\xi):=f_{\xi}^{P}(p)
$$

Observe that if $\tilde{\xi}^{M} \in \operatorname{ker} \Omega$ then $f_{\xi}^{M}$ is constant and therefore $f_{\xi}^{P} \equiv \eta$ is a constraint for $M$ in $P$; whereas if $\tilde{\xi}^{M} \notin \operatorname{ker} \Omega$ then $f_{\xi}^{M}$ is a constraint for $\mathcal{J}^{-1}(\mu)$ in $M$. Thus $\mathcal{J}=\mathrm{J} \circ j_{M}$ and $\mathcal{J}^{-1}(\mu)=\mathrm{J}^{-1}(\mu)$ (see the proof of theorem 6).

It is important to point out that, in general, $\mathrm{J}$ is not strictly speaking a momentum map, because the action $\Psi$ is not necessarily presymplectic for $(P, \omega)$.

In any case, if the assumption 2 is assumed for $(M, \Omega)$, the reduction program follows in the same way as in the case of compatible presymplectic dynamical systems.

\section{Examples}

\subsection{Reduction of non-autonomous systems with symmetry in the presymplectic formulation}

Non-autonomous dynamical systems can be geometrically treated in several ways (see, for instance, [1], [18], [20], 27], [30], [47] for a review on these formulations). Reduction of time-dependent systems with symmetry can be achieved by using the extended or symplectic formulation, and then by using, then, the usual reduction theory for symplectic systems with symmetry (see, for instance, [9] and [33]). Nevertheless, we will use reduction for presymplectic systems, (whose features we have just presented) because it has some advantages in relation to the symplectic case; for instance, singular time-dependent systems with symmetry can be treated in this formulation in a very natural way.

Thus we need to use the presymplectic formulation of non-autonomous systems [15], [20]. The main characteristics of this formulation are the following: the dynamics takes place in a differentiable manifold $P \times \mathbf{R}$, where $\left(P, \omega_{P}\right)$ is either a symplectic manifold, if the non-autonomous system is regular, or a presymplectic one, if it is singular; and we have the natural projections

$$
\tau: P \times \mathbf{R} \rightarrow P \quad ; \quad t: P \times \mathbf{R} \rightarrow \mathbf{R}
$$

The dynamical information is entirely contained in a function $h \in \mathrm{C}^{\infty}(P \times \mathbf{R})$. Then, $P \times \mathbf{R}$ is endowed with the following presymplectic structure

$$
\Omega_{h}:=\tau^{*} \omega_{P}+\mathrm{d} h \wedge \mathrm{d} t
$$

which is exact if, and only if, so is $\omega_{P}$. So, we have the dynamics fully included in the geometry and, therefore, we can obtain the equations of motion stating that $\left(P \times \mathbf{R}, \Omega_{h}, 0\right)$ is a presymplectic Hamiltonian system; that is, in the presymplectic equations of motion $i(X) \Omega_{h}=\mathrm{dH}$, we take $\mathrm{H}=0$. Then the equations of motion are reduced to

$$
i(X) \Omega_{h}=0, X \in \mathcal{X}(P \times \mathbf{R})
$$

Since $\mathrm{dH}=0$, these equations are compatible in $P \times \mathbf{R}$ and, consequently, there exists solution $X \in \operatorname{ker} \Omega_{h}$. On the other hand, if we want to yield the time-reparametrization $t=s$ we must add 
the equation $i(X) \mathrm{d} t=1$; (however, other possible reparametrizations having physical sense are also possible [5], [16]).

We differentiate the following situations:

- The Lagrangian formalism of non-autonomous systems:

$P$ is the tangent bundle $\mathrm{T} Q$ of the configuration space $Q$. Then, given a time-dependent Lagrangian function $\mathcal{L} \in \mathrm{C}^{\infty}(\mathrm{T} Q \times \mathbf{R})$, using the extensions to $\mathrm{T} Q \times \mathbf{R}$ of the natural geometric structures in the tangent bundle (the vertical endomorphism and the Liouville's vector field), we can construct the exact form $\omega_{\mathcal{L}} \in \Omega^{2}(\mathrm{~T} Q \times \mathbf{R})$, which plays the role of the form $\tau^{*} \omega_{P}$, and the energy Lagrangian function $\mathrm{E}_{\mathcal{L}} \in \mathrm{C}^{\infty}(\mathrm{T} Q)$ which plays the role of $h$ in this formalism. Then

$$
\Omega_{h} \equiv \Omega_{\mathcal{L}}=\omega_{\mathcal{L}}+\mathrm{dE}_{\mathcal{L}} \wedge \mathrm{d} t
$$

(see [20] and 21] for a discussion on the construction of these elements).

If the system is regular, then the form $\omega_{\mathcal{L}}$ is symplectic. If the system is singular then $\omega_{\mathcal{L}}$ is a presymplectic form.

- The Hamiltonian formalism of non-autonomous systems:

If the system is not singular, then $\left(P, \omega_{P}\right)$ is a symplectic manifold, $P$ being the cotangent bundle $\mathrm{T}^{*} Q$ of the configuration space $Q$ (if it is hiper-regular) or an open submanifold of it (if it is regular) and $\omega_{P} \equiv \omega \in \Omega^{2}\left(\mathrm{~T}^{*} Q\right)$ being its natural canonical form, which is an exact form. Then, $h \in \mathrm{C}^{\infty}\left(\mathrm{T}^{*} Q\right)$ is the time-dependent Hamiltonian function.

If the system is singular, then $\left(P, \omega_{P}\right)$ is a presymplectic manifold, $j: P \hookrightarrow \mathrm{T}^{*} Q$ being a submanifold of the cotangent bundle $\mathrm{T}^{*} Q$ of the configuration space $Q$ (really it is the image of $\mathrm{T} Q$ by the Legendre transformation) and $\omega_{P}=j^{*} \omega$. Then, $h \in \mathrm{C}^{\infty}\left(\mathrm{T}^{*} Q\right)$ is called the canonical time-dependent Hamiltonian function.

Concerning the study of symmetries, time-dependent dynamical systems display some particular characteristics which are interesting to point out. Thus, from the geometrical and the dynamical point of view, a natural way of defining the concept of symmetry is the following:

Definition 6 Let $G$ be a Lie group, $\left(P \times \mathbf{R}, \Omega_{h}\right)$ a non-autonomous system and $\Phi: G \times(P \times \mathbf{R}) \rightarrow$ $P \times \mathbf{R}$ an action of $G$ on $P \times \mathbf{R}$. $G$ is said to be a group of standard symmetries of this system iff, for every $g \in G$,

1. $\Phi_{g}$ preserves the forms $\tau^{*} \omega_{P}$ and $\mathrm{d} t$; that is,

$$
\Phi_{g}^{*} \tau^{*} \omega_{P}=\tau^{*} \omega_{P} \quad ; \quad \Phi_{g}^{*} \mathrm{~d} t=\mathrm{d} t
$$

2. $\Phi_{g}$ preserves the dynamical function $h$; that is, $\Phi_{g}^{*} h=h$.

The diffeomorphisms $\Phi_{g}$ are called standard symmetries of the system.

The first part of this definition is equivalent to that of cosymplectic action introduced in [33] and agrees also with the concept of standard canonical transformation for time-dependent Hamiltonian systems, which other authors have previously introduced see [5], [16].

As immediate consequences of this definition we have that: 
- If $G$ is a group of standard symmetries of the non-autonomous system $\left(P \times \mathbf{R}, \Omega_{h}\right)$ then, for every $g \in G$, every standard symmetry $\Phi_{g}$ preserves the form $\Omega_{h}$; that is, $\Phi_{g}^{*} \Omega_{h}=\Omega_{h}$.

- $G$ is a group of standard symmetries of the non-autonomous system $\left(P \times \mathbf{R}, \Omega_{h}\right)$ if, and only if, the following three conditions hold for every $\xi \in \mathbf{g}$ :

$$
\mathrm{L}(\tilde{\xi})\left(\tau^{*} \omega_{P}\right)=0
$$

$$
\mathrm{L}(\tilde{\xi}) \mathrm{d} t=0
$$

$$
\mathrm{L}(\tilde{\xi}) h=0
$$

- If $G$ is a group of standard symmetries for the non-autonomous system $\left(P \times \mathbf{R}, \Omega_{h}\right)$ then it is also a symmetry group for the presymplectic Hamiltonian system $\left(P \times \mathbf{R}, \Omega_{h}, 0\right)$ (in the sense of definition (1).

At this point, reduction of non-autonomous dynamical systems with symmetry (both in the Lagrangian or in the Hamiltonian formalism) is merely a direct application of the considerations we made above in order to reduce presymplectic systems with symmetry.

\subsection{Autonomous dynamical systems}

As an example, we are going to analyze the time-independent dynamical systems as the particular case of non-autonomous regular systems which are invariant under time-translations. This study is identical for the Lagrangian and the Hamiltonian formalism and we will do it in general.

Let $\left(P \times \mathbf{R}, \Omega_{h}\right)$ be a non-autonomous regular dynamical system (then $P$ is either $\mathrm{T}^{*} Q$ or $\mathrm{T} Q$ and $\operatorname{dim} P=2 r)$. $G$ is the group of translations in time. The action $\Phi: G \times(P \times \mathbf{R}) \rightarrow P \times \mathbf{R}$ is effective, free and proper. The real Lie algebra $\mathbf{g}$ is spanned by the vector field $\xi \equiv \frac{\partial}{\partial t}$ and hence $\mathbf{g}^{*}=\{\mathrm{d} t\}$. Thus, the set of fundamental vector fields $\tilde{\mathbf{g}}$ is generated by the vector field $\tilde{\xi} \equiv \frac{\partial}{\partial t}$.

Suppose that the dynamical function $h$ is time-independent, that is,

$$
\mathrm{L}\left(\frac{\partial}{\partial t}\right) h=\frac{\partial h}{\partial t}=0
$$

It is evident that this action verifies the conditions of definition 6, and hence $G$ is a symmetry group for this system. Then the action is presymplectic: $\mathrm{L}\left(\frac{\partial}{\partial t}\right) \Omega_{h}=0$, and, in addition, it is strongly presymplectic since the fundamental vector field is Hamiltonian (it is in fact an exact presymplectic action) and

$$
i\left(\frac{\partial}{\partial t}\right) \Omega_{h}=\mathrm{d} h
$$

In this way the momentum map is given by

$$
(\mathcal{J}(x))\left(\frac{\partial}{\partial t}\right):=h(x) \quad(\text { for every } x \in P \times \mathbf{R})
$$

and the level sets of this map, for every weakly regular value $\mu=\mu_{0} \mathrm{~d} t \in \mathbf{g}^{*}$, are

$$
\mathcal{J}^{-1}(\mu):=\left\{x \in P \times \mathbf{R} \mid h(x)=\mu_{0}\right\}
$$

(they are defined by the constraints $\zeta:=h-\mu_{0}$ and hence $\frac{\partial}{\partial t}$ is tangent to all of them). They are the hypersurfaces of constant energy in $P \times \mathbf{R}$. 
In each one, we have the presymplectic Hamiltonian system $\left(\mathcal{J}^{-1}(\mu), \Omega_{\mu}, 0\right)$, where $\Omega_{\mu}:=$ $j_{\mu}^{*} \Omega_{h}=j_{\mu}^{*} \tau^{*} \Omega_{P}$. Notice that, even though $\operatorname{dim} \mathcal{J}^{-1}(\mu)$ is even, $\Omega_{\mu}$ is presymplectic since ker $\Omega_{\mu}=$ $\left\{\frac{\partial}{\partial t}, X_{\mu}\right\}$, where $X_{\mu} \in \mathcal{X}\left(\mathcal{J}^{-1}(\mu)\right)$ is the solution of the dynamical equation $i\left(X_{\mu}\right) \Omega_{\mu}=0$. So, in this case, since $G=G_{\mu}, \tilde{\mathbf{g}}_{\mu}$ is generated by the vector field $\tilde{\xi} \equiv \frac{\partial}{\partial t}$ and we have that $\tilde{\mathbf{g}}_{\mu} \subset$ ker $\Omega_{\mu}$. Therefore, applying the reduction theorems, we have the same situation as the first item in theorem 2, and hence this presymplectic system reduces to another one $\left(\mathcal{J}^{-1}(\mu) / G, \hat{\Omega}, 0\right)$. This is a $(2 r-1)$ dimensional differentiable manifold (and then $\hat{\Omega}$ is a presymplectic form with $\operatorname{rank} \hat{\Omega}=2 r-2$ ) where the global coordinate $t$ is avoided. The evolution equations are

$$
i(\hat{X}) \hat{\Omega}=0 \quad, \quad \tilde{X} \in \mathcal{X}\left(\mathcal{J}^{-1}(\mu) / G\right)
$$

Observe that the main advantage of this reduction procedure is that, in addition to eliminating the ignorable time-coordinate, it already gives the solution of dynamics directly on the corresponding hypersurface of constant energy. This is an advance in relation to the use of the symplectic reduction procedure of Marsden and Weinstein, applied for treating this same example but starting from the extended symplectic formalism of the non-autonomous systems. In this case, even though the reduced dynamical system is regular (and then symplectic), the symplectic reduction procedure removes time only (from the initial time-dependent system), but it does not give the dynamics on the constant-energy hypersurfaces which is obtained by projection, that is, after another step not included in the reduction procedure (see [33]).

Nevertheless, a further reduction could be made by the residual part of ker $\Omega_{\mu}$ (that is those one generated by $X_{\mu}$ ) or, what is equivalent, make the reduction of the presymplectic Hamiltonian system $\left(\mathcal{J}^{-1}(\mu), \Omega_{\mu}, 0\right)$ by ker $\Omega_{\mu}$. In this way we would have the situation of the second item in theorem 2 , and hence this presymplectic system reduces to $\left(\mathcal{J}^{-1}(\mu) / \operatorname{ker} \Omega_{\mu}, \hat{\Omega}^{\prime}, 0\right)$, where $\mathcal{J}^{-1}(\mu) / \operatorname{ker} \Omega_{\mu}$ is a $(2 r-2)$-dimensional differentiable manifold and $\hat{\Omega}^{\prime}$ is a symplectic form. As a consequence, there is no dynamics in this reduced system; that is, the orbit space is made of the dynamical trajectories of the initial time-independent dynamical system (for a fixed constant value of the energy).

\subsection{A mechanical model of field theories: description}

The following example we study is based in a mechanical model of field theories (coupled to external fields) due to Capri and Kobayashi [11], [12]. See also [17] for a deeper analysis.

The general form of the Lagrangian of the system is

$$
\mathcal{L}=\dot{\psi}^{* a} m_{a b} \dot{\psi}^{b}+\dot{\psi}^{* a} c_{a b} \psi^{b}-\psi^{* a} \bar{c}_{a b} \dot{\psi}^{b}-\psi^{* a} r_{a b} \psi^{b}
$$

where:

- $\psi^{a}, \psi^{* b}(a, b=1, \ldots, n)$ are scalar (complex) fields. In this mechanical model they will be interpreted as independent "coordinates" of certain $2 n$-dimensional configuration space $Q$.

- $m_{a b}, c_{a b}, \bar{c}_{a b}, r_{a b}$ are (time-independent) functional coefficients such that, in order $\mathcal{L}$ to be real, the matrices $m_{a b}, r_{a b}$ are hermitian and $\bar{c}_{a b}^{*}=-c_{b a}$. In particular, if rank $\left(m_{a b}\right)<n$, the Lagrangian is singular and this is the case of greatest interest to us.

Lagrangians of this kind enables us to describe some relativistic bosonic field theories (after a $(3+1)$ decomposition), where the eventual coupling to external fields is tucked away in the coefficients $c_{a b}$ and $r_{a b}$. 
In order to make the example more pedagogical, we will analyze the following simple case: $a, b=1,2,3$ and

$$
m_{a b}=\left(\begin{array}{ccc}
0 & 0 & 0 \\
0 & m_{2} & 0 \\
0 & 0 & m_{3}
\end{array}\right) \quad c_{a b}=\bar{c}_{a b}=\frac{1}{2}\left(\begin{array}{ccc}
0 & 0 & 0 \\
0 & i & 0 \\
0 & 0 & i
\end{array}\right) \quad r_{a b}=\left(\begin{array}{ccc}
1 & 0 & 0 \\
0 & 1 & 0 \\
0 & 0 & 1
\end{array}\right)
$$

We can write the Lagrangian in its real form by the change

$$
\begin{array}{cccccrl}
\psi^{1}=x^{1}+i y^{1} & , & \psi^{2}=x^{2}+i y^{2} & , & \psi^{3}=x^{3}+i y^{3} \\
\dot{\psi}^{1}=u^{1}+i v^{1} & , & \dot{\psi}^{2}=u^{2}+i v^{2} & , & \dot{\psi}^{3}=u^{3}+i v^{3} \\
\dot{\psi}^{* 1}=x^{1}-i y^{1} & , & \psi^{* 2}=x^{2}-i y^{2} & , & \psi^{* 3}=x^{3}-i y^{3} \\
\dot{\psi}^{* 1}=u^{1}-i v^{1} & , & \dot{\psi}^{* 2}=u^{2}-i v^{2} & , & \dot{\psi}^{* 3}=u^{3}-i v^{3}
\end{array}
$$

and hence

$$
\begin{aligned}
\mathcal{L}= & m_{2}\left(\left(u^{2}\right)^{2}+\left(v^{2}\right)^{2}\right)+m_{3}\left(\left(u^{3}\right)^{2}+\left(v^{3}\right)^{2}\right)+v^{2} x^{2}-u^{2} y^{2}+v^{3} x^{3}-u^{3} y^{3} \\
& -\left(x^{1}\right)^{2}-\left(y^{1}\right)^{2}-\left(x^{2}\right)^{2}-\left(y^{2}\right)^{2}-\left(x^{3}\right)^{2}-\left(y^{3}\right)^{2}
\end{aligned}
$$

Here, the configuration space is taken to be $Q=\mathbf{R}^{6}$ with local coordinates $\left(x^{i}, y^{i}\right)(i=1,2,3)$ and $\mathrm{T} Q \simeq \mathbf{R}^{12}$ with local coordinates $\left(x^{i}, y^{i} ; u^{i}, v^{i}\right)$, where $u^{i}, v^{i}$ denote the generalized velocities corresponding to $x^{i}, y^{i}$ respectively. Using the canonical structures of the tangent bundle TQ, the Lagrangian 2-form and the energy Lagrangian function are constructed:

$$
\begin{aligned}
& \omega_{\mathcal{L}}=2\left[m_{2}\left(\mathrm{~d} x^{2} \wedge \mathrm{d} u^{2}+\mathrm{d} y^{2} \wedge \mathrm{d} v^{2}\right)+m_{3}\left(\mathrm{~d} x^{3} \wedge \mathrm{d} u^{3}+\mathrm{d} y^{3} \wedge \mathrm{d} v^{3}\right)+\mathrm{d} x^{2} \wedge \mathrm{d} y^{2}+\mathrm{d} x^{3} \wedge \mathrm{d} y^{3}\right] \\
& \mathrm{E}_{\mathcal{L}}=m_{2}\left(\left(u^{2}\right)^{2}+\left(v^{2}\right)^{2}\right)+m_{3}\left(\left(u^{3}\right)^{2}+\left(v^{3}\right)^{2}\right)+\left(x^{1}\right)^{2}+\left(y^{1}\right)^{2}+\left(x^{2}\right)^{2}+\left(y^{2}\right)^{2}+\left(x^{3}\right)^{2}+\left(y^{3}\right)^{2}
\end{aligned}
$$

The system is singular since $\omega_{\mathcal{L}}$ is presymplectic and

$$
\operatorname{ker} \omega_{\mathcal{L}} \equiv\left\{\frac{\partial}{\partial x^{1}}, \frac{\partial}{\partial y^{1}}, \frac{\partial}{\partial u^{1}}, \frac{\partial}{\partial v^{1}}\right\}
$$

$\left(\mathrm{T} Q, \omega_{\mathcal{L}}, \mathrm{E}_{\mathcal{L}}\right)$ is a presymplectic dynamical system which is not compatible since

$$
i\left(\frac{\partial}{\partial x^{1}}\right) \mathrm{dE}_{\mathcal{L}}=2 x^{1} \neq 0 \quad, \quad i\left(\frac{\partial}{\partial y^{1}}\right) \mathrm{dE}_{\mathcal{L}}=2 y^{1} \neq 0
$$

So the constraints

$$
\eta_{1}:=x^{1}=0 \quad, \quad \eta_{2}:=y^{1}=0
$$

define locally a submanifold $j_{M}: M \hookrightarrow \mathrm{T} Q$ where the vector fields which are solutions of the dynamical equation

$$
\left.\left(i(X) \omega_{\mathcal{L}}-\mathrm{dE}_{\mathcal{L}}\right)\right|_{M}=0
$$

are the following

$$
\begin{aligned}
\left.X\right|_{M}= & f^{1} \frac{\partial}{\partial x^{1}}+u^{2} \frac{\partial}{\partial x^{2}}+u^{3} \frac{\partial}{\partial x^{3}}+g^{1} \frac{\partial}{\partial y^{1}}+v^{2} \frac{\partial}{\partial y^{2}}+v^{3} \frac{\partial}{\partial y^{3}}+F^{1} \frac{\partial}{\partial u^{1}}-\frac{1}{m_{2}}\left(v^{2}+x^{2}\right) \frac{\partial}{\partial u^{2}} \\
& -\frac{1}{m_{3}}\left(v^{3}+x^{3}\right) \frac{\partial}{\partial u^{3}}+G^{1} \frac{\partial}{\partial v^{1}}+\frac{1}{m_{2}}\left(u^{2}-y^{2}\right) \frac{\partial}{\partial v^{2}}+\frac{1}{m_{3}}\left(u^{3}-y^{3}\right) \frac{\partial}{\partial v^{3}}
\end{aligned}
$$

where $f^{1}, g^{1}, F^{1}, G^{1}$ are arbitrary functions. Now we consider two options:

1. If we look for solutions of the dynamics which are second order differential equations (SODE) then, in this case, we obtain such a solution taking the first two arbitrary functions to be $f^{1}=u^{1}$ and $g^{1}=v^{1}$. Therefore the stability of this vector field on the constraints $\eta_{1}, \eta_{2}$ 
originates two new constraints (which are called non-dynamical constraints following the terminology of [44])

$$
\chi_{1}:=u^{1}=0 \quad, \quad \chi_{2}:=v^{1}=0
$$

which, joined to the above ones $\eta_{1}, \eta_{2}$, define locally the submanifold $j_{S}: S \hookrightarrow \mathrm{T} Q$. Finally, the stability of the SODE vector field on the last constraints fixes the value of the remaining arbitrary functions to be $F^{1}=0, G^{1}=0$. So the final constraint submanifold is $S$ (local coordinates are $\left(x^{2}, x^{3}, y^{2}, y^{3}, u^{2}, u^{3}, v^{2}, v^{3}\right)$ and

$$
\begin{aligned}
\Omega_{S}:=j_{S}^{*} \omega_{\mathcal{L}}= & 2\left[m_{2}\left(\mathrm{~d} x^{2} \wedge \mathrm{d} u^{2}+\mathrm{d} y^{2} \wedge \mathrm{d} v^{2}\right)+m_{3}\left(\mathrm{~d} x^{3} \wedge \mathrm{d} u^{3}+\mathrm{d} y^{3} \wedge \mathrm{d} v^{3}\right)\right. \\
& \left.+\mathrm{d} x^{2} \wedge \mathrm{d} y^{2}+\mathrm{d} x^{3} \wedge \mathrm{d} y^{3}\right] \\
\mathrm{E}_{S}:=j_{S}^{*} \mathrm{E}_{\mathcal{L}}= & m_{2}\left(\left(u^{2}\right)^{2}+\left(v^{2}\right)^{2}\right)+m_{3}\left(\left(u^{3}\right)^{2}+\left(v^{3}\right)^{2}\right)+\left(x^{2}\right)^{2}+\left(y^{2}\right)^{2}+\left(x^{3}\right)^{2}+\left(y^{3}\right)^{2}
\end{aligned}
$$

Observe that, in this example, $\left(S, \Omega_{S}\right)$ is a symplectic manifold. The SODE vector field tangent to $S$ being the (unique) solution of the dynamical equation

$$
\left.\left(i(X) \omega_{\mathcal{L}}-\mathrm{dE}_{\mathcal{L}}\right)\right|_{S}=0
$$

is then

$$
\begin{aligned}
\left.X\right|_{S}= & u^{2} \frac{\partial}{\partial x^{2}}+u^{3} \frac{\partial}{\partial x^{3}}+v^{2} \frac{\partial}{\partial y^{2}}+v^{3} \frac{\partial}{\partial y^{3}}-\frac{1}{m_{2}}\left(v^{2}+x^{2}\right) \frac{\partial}{\partial u^{2}} \\
& -\frac{1}{m_{3}}\left(v^{3}+x^{3}\right) \frac{\partial}{\partial u^{3}}+\frac{1}{m_{2}}\left(u^{2}-y^{2}\right) \frac{\partial}{\partial v^{2}}+\frac{1}{m_{3}}\left(u^{3}-y^{3}\right) \frac{\partial}{\partial v^{3}}
\end{aligned}
$$

(See also 17] for a more detailed discussion on this analysis).

2. If we look for solutions of the dynamics which are not SODE, then the stability of (15) on the constraints $\eta_{1}, \eta_{2}$ fixes the value of the first two arbitrary functions to be $f^{1}=0, g^{1}=0$. So the final constraint submanifold is $M$ (local coordinates are $\left(x^{2}, x^{3}, y^{2}, y^{3}, u^{1}, u^{2}, u^{3}, v^{1}, v^{2}, v^{3}\right)$

and the coordinate expressions of $\Omega_{M}:=j_{M}^{*} \omega_{\mathcal{L}}$ and $\mathrm{E}_{M}:=j_{M}^{*} \mathrm{E}_{\mathcal{L}}$ are the same as for $\Omega_{S}$ and $\mathrm{E}_{S}$ respectively. Hence $\left(M, \Omega_{M}\right)$ is a presymplectic manifold with

$$
\text { ker } \Omega_{M} \equiv\left\{\frac{\partial}{\partial u^{1}}, \frac{\partial}{\partial v^{1}}\right\}
$$

and the vector fields tangent to $M$ being solutions of the dynamical equation (14) are

$$
\begin{aligned}
\left.X\right|_{M}= & u^{2} \frac{\partial}{\partial x^{2}}+u^{3} \frac{\partial}{\partial x^{3}}+v^{2} \frac{\partial}{\partial y^{2}}+v^{3} \frac{\partial}{\partial y^{3}}+F^{1} \frac{\partial}{\partial u^{1}}-\frac{1}{m_{2}}\left(v^{2}+x^{2}\right) \frac{\partial}{\partial u^{2}} \\
& -\frac{1}{m_{3}}\left(v^{3}+x^{3}\right) \frac{\partial}{\partial u^{3}}+G^{1} \frac{\partial}{\partial v^{1}}+\frac{1}{m_{2}}\left(u^{2}-y^{2}\right) \frac{\partial}{\partial v^{2}}+\frac{1}{m_{3}}\left(u^{3}-y^{3}\right) \frac{\partial}{\partial v^{3}}
\end{aligned}
$$

\subsection{A mechanical model of field theories: symmetries and reduction}

Next we are going to study the symmetries of the systems, splitting the two cases considered in the above section; that is, we will apply the reduction procedure to the compatible dynamical systems $\left(S, \Omega_{S}, \mathrm{E}_{S}\right)$ and $\left(M, \Omega_{M}, \mathrm{E}_{M}\right)$.

Both of them exhibit some non-gauge rigid symmetries which are rotations on $Q$ whose infinitesimal generators are the following vector fields in $Q$

$$
x^{2} \frac{\partial}{\partial y^{2}}-y^{2} \frac{\partial}{\partial x^{2}} \quad, \quad x^{3} \frac{\partial}{\partial y^{3}}-y^{3} \frac{\partial}{\partial x^{3}}
$$


and whose canonical liftings to $\mathrm{T} Q$ give the following fundamental vector fields

$$
\begin{aligned}
& \tilde{\xi}_{1}=x^{2} \frac{\partial}{\partial y^{2}}-y^{2} \frac{\partial}{\partial x^{2}}+u^{2} \frac{\partial}{\partial v^{2}}-v^{2} \frac{\partial}{\partial u^{2}} \\
& \tilde{\xi}_{2}=x^{3} \frac{\partial}{\partial y^{3}}-y^{3} \frac{\partial}{\partial x^{3}}+u^{3} \frac{\partial}{\partial v^{3}}-v^{3} \frac{\partial}{\partial u^{3}}
\end{aligned}
$$

In fact, these vector fields generate infinitesimal symmetries for these presymplectic systems because both of them are tangent to $S$ and $M$ and they satisfy that

$$
\begin{aligned}
j_{M}^{*} \mathrm{~L}\left(\tilde{\xi}_{k}\right) \mathrm{E}_{\mathcal{L}} & =0=j_{S}^{*} \mathrm{~L}\left(\tilde{\xi}_{k}\right) \mathrm{E}_{\mathcal{L}} \\
j_{M}^{*} \mathrm{~L}\left(\tilde{\xi}_{k}\right) \omega_{\mathcal{L}} & =0=j_{S}^{*} \mathrm{~L}\left(\tilde{\xi}_{k}\right) \omega_{\mathcal{L}}
\end{aligned}
$$

since $\mathrm{L}\left(\tilde{\xi}_{k}\right) \mathrm{E}_{\mathcal{L}}=0(k=1,2)$ and $\mathrm{L}\left(\tilde{\xi}_{k}\right) \omega_{\mathcal{L}}=0$, so both of them are presymplectic Hamiltonian vector fields in $\left(S, \Omega_{S}, \mathrm{E}_{S}\right)$ and $\left(M, \Omega_{M}, \mathrm{E}_{M}\right)$.

\section{Reduction of the system $\left(S, \Omega_{S}, \mathrm{E}_{S}\right)$.}

Since $\left(S, \Omega_{S}\right)$ is a symplectic manifold, there are no gauge symmetries and the only symmetries to be taken into account are the rigid ones which have just been introduced. Denoting by $G$ the corresponding group and by $\mathbf{g}$ its Lie algebra, then $\tilde{\mathbf{g}} \equiv\left(\tilde{\xi}^{1}, \tilde{\xi}_{2}\right)$. The action considered is in fact strongly presymplectic, since it is an exact action in relation to the 1-form

$$
j_{S}^{*} \theta_{\mathcal{L}}=\left(2 m_{2} u^{2}+y^{2}\right) \mathrm{d} x^{2}+\left(2 m_{2} v^{2}-x^{2}\right) \mathrm{d} y^{2}+\left(2 m_{3} u^{3}-y^{3}\right) \mathrm{d} x^{3}+\left(2 m_{3} v^{3}+x^{3}\right) \mathrm{d} y^{3}
$$

The presymplectic Hamiltonian functions of $\tilde{\xi}^{1}$ and $\tilde{\xi}^{2}$ in $\left(S, \Omega_{S}\right)$ are

$$
f_{\xi_{1}}=2 m_{2}\left(x^{2} v^{2}-y^{2} u^{2}\right)-\left(x^{2}\right)^{2}-\left(y^{2}\right)^{2} \quad, \quad f_{\xi_{2}}=2 m_{3}\left(x^{3} v^{3}-y^{3} u^{3}\right)-\left(x^{3}\right)^{2}-\left(y^{3}\right)^{2}
$$

So a momentum map $\mathcal{J}_{S}$ can be defined for this action and, taking into account the discussion in the section 3.5, for every weakly regular value $\mu \equiv\left(\mu_{1}, \mu_{2}\right) \in \mathbf{g}^{*}$, its level sets $\mathcal{J}_{S}^{-1}(\mu)$ are defined as submanifolds of $\mathrm{T} Q$ by the constraints

$$
\begin{aligned}
\eta_{1}:=x^{1}=0 \quad, \quad \eta_{2}:=y^{1}=0 \quad, \quad \chi_{1}:=u^{1}=0 \quad, \quad \chi_{2}:=v^{1}=0 \\
f_{\xi_{1}}:=2 m_{2}\left(x^{2} v^{2}-y^{2} u^{2}\right)-\left(x^{2}\right)^{2}-\left(y^{2}\right)^{2}=\mu_{1} \\
f_{\xi_{2}}:=2 m_{3}\left(x^{3} v^{3}-y^{3} u^{3}\right)-\left(x^{3}\right)^{2}-\left(y^{3}\right)^{2}=\mu_{2}
\end{aligned}
$$

The submanifolds $\left(\mathcal{J}_{S}^{-1}(\mu), \Omega_{S_{\mu}}\right)$ are presymplectic and 6-dimensional. Next, the final step of the reduction procedure leads to the 4 -dimensional quotient manifolds $\left(\mathcal{J}_{S}^{-1}(\mu) / \operatorname{ker} \Omega_{S_{\mu}}, \hat{\Omega}_{S}\right)$.

2. Reduction of the system $\left(M, \Omega_{M}, \mathrm{E}_{M}\right)$.

This compatible presymplectic system exhibits the above rigid symmetries as well as gauge symmetries, which are infinitesimally generated by the fundamental vector fields

$$
\tilde{\xi}_{3}=\frac{\partial}{\partial u_{1}} \quad, \quad \tilde{\xi}_{4}=\frac{\partial}{\partial v_{1}}
$$

(which generate ker $\Omega_{M}$ ). Let $G$ be the group of all these symmetries and $\mathbf{g}$ its Lie algebra, then $\tilde{\mathbf{g}} \equiv\left(\tilde{\xi}_{1}, \tilde{\xi}_{2}, \tilde{\xi}_{3}, \tilde{\xi}_{4}\right)$. The action considered is in fact also strongly presymplectic, since it is an exact action in relation to the 1 -form $j_{M}^{*} \theta_{\mathcal{L}}$. The presymplectic Hamiltonian functions of the fundamental vector fields in $\left(S, \Omega_{S}\right)$ are

$$
\begin{aligned}
& f_{\xi_{1}}:=2 m_{2}\left(x^{2} v^{2}-y^{2} u^{2}\right)-\left(x^{2}\right)^{2}-\left(y^{2}\right)^{2} \quad, \quad f_{\xi_{2}}:=2 m_{3}\left(x^{3} v^{3}-y^{3} u^{3}\right)-\left(x^{3}\right)^{2}-\left(y^{3}\right)^{2} \\
& f_{\xi_{3}}:=0 \quad, \quad f_{\xi_{4}}:=0
\end{aligned}
$$


where the constant value of $f_{\xi_{3}}$ and $f_{\xi_{4}}$ equal to 0 is just a possible choice for the constant Hamiltonian functions corresponding to the vector fields $\tilde{\xi}_{3}$ and $\tilde{\xi}_{4}$ respectively. So a momentum map $\mathcal{J}_{M}$ can be defined for this action and, taking into account the discussion in the section 3.5, for weakly regular values $\mu \equiv\left(\mu_{1}, \mu_{2}, 0,0\right) \in \mathbf{g}^{*}$, its level sets $\mathcal{J}_{M}^{-1}(\mu)$ are defined as submanifolds of $\mathrm{T} Q$ by the constraints

$$
\begin{gathered}
f_{\xi_{1}}:=2 m_{2}\left(x^{2} v^{2}-y^{2} u^{2}\right)-\left(x^{2}\right)^{2}-\left(y^{2}\right)^{2}=\mu_{1} \\
f_{\xi_{2}}:=2 m_{3}\left(x^{3} v^{3}-y^{3} u^{3}\right)-\left(x^{3}\right)^{2}-\left(y^{3}\right)^{2}=\mu_{2} \\
\eta_{1}:=x^{1}=0 \quad, \quad \eta_{2}:=y^{1}=0
\end{gathered}
$$

(Observe that $\eta_{1}:=x^{1}$ and $\eta_{2}:=y^{1}$ are the presymplectic Hamiltonian functions of $\tilde{\xi}_{3}$ and $\tilde{\xi}_{4}$ in $\left(\mathrm{T} Q, \Omega_{\mathcal{L}}\right)$, respectively). Now, the submanifolds $\left(\mathcal{J}_{M}^{-1}(\mu), \Omega_{M_{\mu}}\right)$ are presymplectic and 8-dimensional and the final quotient manifolds $\left(\mathcal{J}_{M}^{-1}(\mu) / \operatorname{ker} \Omega_{M_{\mu}}, \hat{\Omega}_{M}\right)$ are 4-dimensional.

Nevertheless, this quotient manifold is locally symplectomorphic to $\left(\mathcal{J}_{S}^{-1}(\mu) / \operatorname{ker} \Omega_{S_{\mu}}, \hat{\Omega}_{S}\right)$. In fact; instead of using the complete presymplectic reduction, we can apply to the system $\left(M, \Omega_{M}, \mathrm{E}_{M}\right)$, first, gauge reduction and, afterwards, standard symplectic reduction, then obtaining a quotient manifold which is symplectomorphic to $\left(\mathcal{J}_{M}^{-1}(\mu) / \operatorname{ker} \Omega_{M_{\mu}}, \hat{\Omega}_{M}\right.$ ) (see section 3.3) and locally symplectomorphic to $\left(\mathcal{J}_{S}^{-1}(\mu) / \operatorname{ker} \Omega_{S_{\mu}}, \hat{\Omega}_{S}\right)$ (obviously).

\section{Comment:}

In this example we have just shown that whether or not non-dynamical constraints (that is, those arising in the stabilization algorithm from demanding that the vector field solution of the Lagrange equations to be a SODE) are taken into account in the reduction procedure is irrelevant, since, in any case, we obtain the same quotient manifold.

In reality this must be a general property. In fact: let $\left(\mathrm{T} Q, \omega_{\mathcal{L}}, \mathrm{E}_{\mathcal{L}}\right)$ be a singular (but almostregular [22], [44]) Lagrangian system, $\left(M, \Omega_{M}\right)$ the final constraint submanifold when the SODEcondition is not considered and $\left(S, \Omega_{S}\right)$ the final constraint submanifold when the $S O D E$-condition is considered, such that we have a group of rigid symmetries both for $\left(M, \Omega_{M}, \mathrm{E}_{M}\right)$ and $\left(S, \Omega_{S}, \mathrm{E}_{S}\right)$. Then, if the assumption 2 holds for $\left(M, \Omega_{M}\right)$ and $\left(S, \Omega_{S}\right)$, the complete presymplectic reduction procedure leads to the same reduced system for both systems.

The reason for this feature lies in the following facts: As is proved in [44, the non-dynamical constraints defining $S$ in $M$ remove degrees of freedom in the leaves of the foliation generated by the vertical part of ker $\Omega_{\mathcal{L}}$ which, on its turn, is included in ker $\Omega_{M}$. As a consequence, it is also proved that ker $\Omega_{S} \subset \operatorname{ker} \Omega_{M}$. But, as assumption 2 holds, the final quotient in the complete presymplectic reduction is made by a foliation whose leaves contain those of ker $\Omega_{M}$ or ker $\Omega_{S}$ respectively in each case. Therefore, when the reduction is made for $\left(M, \Omega_{M}\right)$, the degrees of freedom in the leaves of the foliation generated by the vertical part of ker $\Omega_{\mathcal{L}}$ are removed in the final quotient. However, when the reduction is made for $\left(S, \Omega_{S}\right)$, these degrees of freedom have been previously removed.

\subsection{The conformal particle}

Finally, we consider the system of a massless relativistic particle with conformal symmetry. The original Lagrangian function was introduced by Marnelius [38 and, subsequently, Siegel used it for describing the behaviour of these kind of particles [48]. Recently, Gràcia and Roca [26] have carefully studied the gauge transformations for this system. 
The configuration space of this system is $Q=\mathbf{R}^{d+2} \times \mathbf{R}$ and is locally coordinated by the set $\left(q^{a}, \lambda\right)(a=0,1, \ldots, d+1)$, where $\lambda$ is an unphysical parameter which is introduced in order to make the description of the system covariant, and it is responsible for the local scale invariance. At the Lagrangian level, the system is dynamically described by the Lagrangian function

$$
\mathcal{L}:=\frac{1}{2} g_{a b}\left(v^{a} v^{b}-\lambda q^{a} q^{b}\right) \in \mathrm{C}^{\infty}(\mathrm{T} Q)
$$

where $g$ is an indefinite metric in $\mathbf{R}^{d+2}$ with signature $\operatorname{sign}\left(g_{a b}\right)=(1,-1, \ldots,-1,1)$. From here and using the canonical structures of the tangent bundle $\mathrm{T} Q$, we construct the Lagrangian 2-form and the energy Lagrangian function:

$$
\omega_{\mathcal{L}}=g_{a b} \mathrm{~d} q^{a} \wedge \mathrm{d} v^{b} \quad ; \quad \mathrm{E}_{\mathcal{L}}=\frac{1}{2} g_{a b}\left(v^{a} v^{b}+\lambda q^{a} q^{b}\right)
$$

( $v^{a}$ denote the generalized velocities corresponding to the coordinates $q^{a}$ ). The system is singular since the generalized velocity $u$ corresponding to the generalized coordinate $\lambda$ does not appear explicitly in the Lagrangian function. Hence $\omega_{\mathcal{L}}$ is presymplectic and ker $\omega_{\mathcal{L}} \equiv\left\{\frac{\partial}{\partial \lambda}, \frac{\partial}{\partial u}\right\}$. So $\left(\mathrm{T} Q, \omega_{\mathcal{L}}, \mathrm{E}_{\mathcal{L}}\right)$ is a presymplectic dynamical system which is not compatible since $i\left(\frac{\partial}{\partial \lambda}\right) \mathrm{d} \mathrm{E}_{\mathcal{L}} \neq 0$ . Using some of the known stabilization algorithms, we find that the final constraint submanifold $j_{M}: M \hookrightarrow \mathrm{T} Q$ is defined by the constraints [26]

$$
\eta_{1}=\frac{1}{2} g_{a b} q^{a} q^{b}, \eta_{2}=g_{a b} v^{a} q^{b}, \eta_{3}=g_{a b} v^{a} v^{b}-\lambda g_{a b} q^{a} q^{b}
$$

In this case we can take a basis of constraints made of presymplectic Hamiltonian functions, for instance

$$
f_{\xi_{1}}=\frac{1}{2} g_{a b} q^{a} q^{b} \quad, \quad f_{\xi_{2}}=g_{a b} v^{a} q^{b} \quad, \quad f_{\xi_{3}}=\frac{1}{2} g_{a b} v^{a} v^{b}
$$

The vector fields which are solutions of the dynamical equation

$$
\left.\left(i(X) \omega_{\mathcal{L}}-\mathrm{dE}_{\mathcal{L}}\right)\right|_{M}=0
$$

are the following

$$
\left.X\right|_{M}=v^{a} \frac{\partial}{\partial q^{a}}+\lambda q^{a} \frac{\partial}{\partial v^{a}}+u \frac{\partial}{\partial \lambda}+f \frac{\partial}{\partial u}
$$

(where $f$ is an arbitrary function).

The compatible presymplectic system $(M, \Omega, \mathrm{H})=\left(M, j_{M}^{*} \omega_{\mathcal{L}}, j_{M}^{*} \mathrm{E}_{\mathcal{L}}\right)$ exhibits point gauge symmetries which are infinitesimally generated by the following fundamental vector fields

$$
\left(\tilde{\xi}_{1}, \tilde{\xi}_{2}, \tilde{\xi}_{3}, \tilde{\xi}_{4}, \tilde{\xi}_{5}\right)=\left(q^{a} \frac{\partial}{\partial v^{a}}, v^{a} \frac{\partial}{\partial v^{a}}-q^{a} \frac{\partial}{\partial q^{a}}, v^{a} \frac{\partial}{\partial q^{a}}, \frac{\partial}{\partial \lambda}, \frac{\partial}{\partial u}\right)
$$

Observe that $\tilde{\xi}_{1}, \tilde{\xi}_{2}, \tilde{\xi}_{3}$ are the presymplectic Hamiltonian vector fields corresponding to $f_{\xi_{1}}, f_{\xi_{2}}, f_{\xi_{3}}$ respectively and $\tilde{\xi}_{4}, \tilde{\xi}_{5} \in$ ker $\Omega$. They are all tangent to $M$ and, hence, they make a local basis of $\underline{\operatorname{ker} j_{M}^{*} \Omega}$.

It is interesting to note that the system is also invariant under rigid $\mathbf{O}(2, d)$ rotations. Nevertheless, it can be shown that there exist $\mathbf{O}(2, d)$ Lagrangian gauge transformations (see [26] and [38) and hence, in this case, this group of symmetries is a closed subgroup of the gauge group $\mathcal{G}$.

Taking all of this into account, the action of $\mathcal{G}$ on $\left(M, j_{M}^{*} \omega_{\mathcal{L}}\right)$ is strongly presymplectic (it is in fact an exact action in relation to the Lagrangian 1-form $\left.j_{M}^{*} \theta_{\mathcal{L}}=j_{M}^{*}\left(g_{a b} v^{a} \mathrm{~d} q^{b}\right)\right)$. Thus, a momentum map $\mathcal{J}$ can be defined for this action such that $M=\mathcal{J}^{-1}(0)$. Therefore, the presymplectic reduction procedure is simply the well-known gauge reduction for the compatible presymplectic system $\left(M, j_{M}^{*} \omega_{\mathcal{L}}, j_{M}^{*} \mathrm{E}_{\mathcal{L}}\right)$. 


\section{Conclusions and outlook}

We have made a study about actions of Lie groups on presymplectic manifolds and the subsequent reduction procedure. The main results and considerations here discussed are the following:

- We have made the natural extension of the concepts of the theory of symplectic actions of Lie groups on symplectic manifolds to this case.

- The existence of comomentum and momentum maps are analyzed, obtaining an obstruction similar to the symplectic case (but involving the set $B_{h}^{1}(M) / Z_{h}^{1}(M)$ instead of the first cohomology group $\left.H^{1}(M)\right)$.

- We have investigated the properties and characteristics of the level sets of the momentum map for weakly regular values, as a standpoint for reduction. As a particular result, the interpretation of these level sets as the maximal integral submanifolds of a Pfaff system allows us to simplify the proof of some results. We hope that this interpretation will be of interest with a view to extending the reduction procedure to field theories.

- The reduction of presymplectic manifolds by presymplectic actions of Lie groups has been achieved for weakly regular values of the momentum map, following the guidelines of the symplectic reduction theory of Marsden-Weinstein. With the usual hypothesis, the reduced phase space is endowed with a structure of presymplectic manifold, in general.

- The concept of symmetry for presymplectic dynamical systems is displayed. The reduction of compatible and non-compatible presymplectic dynamical systems with symmetry is made as an application of the theory just developed. These results hold both for presymplectic Lagrangian or Hamiltonian systems.

When gauge symmetries are taken together with the non-gauge symmetries of the system, then the reduced phase space is endowed with a structure of symplectic manifold with dynamics of Hamiltonian type.

- The procedure of complete presymplectic reduction allows us to reach the orbit space in a straightforward way, in comparison with other step-by-step reduction procedures, namely, coisotropic imbedding plus symplectic reduction and gauge reduction plus symplectic reduction, which lead to the same final reduced phase space. The equivalence of all these methods is also proved.

- As an example, we have considered non-autonomous dynamical systems. Starting from the presymplectic formulation of these systems (which allow us to include also the singular case in a natural way), we have adapted the notion of symmetry, and then by applying the reduction procedure previously studied, results similar to those of other works that have analyzed this problem have been obtained. The main advantage of the formalism is that the treatment of the singular case is absolutely "on way".

- As a particular case, the reduction of time-dependent regular dynamical systems is considered in the framework of time-invariant non-autonomous systems. In this case, the reduced phase space is a contact manifold since the level sets of the momentum map are the energy constant hypersurfaces and reduction removes the time coordinate from the initial system. In this way, in our opinion, this is a better result than those obtained applying the symplectic reduction techniques to the extended phase space of the system, since reduction then leads to a symplectic system in the reduced phase space, but does not directly give the dynamics on the constant-energy hypersurfaces. 
- Another interesting example is the complete reduction of a particular case of the CapriKobayashi mechanical model for field theories coupled to external fields, exhibiting both gauge and non-gauge symmetries, in the Lagrangian formalism. It is shown that, under suitable circumstances, the existence of Lagrangian constraints arising from the search for dynamical solutions which are second order differential equations is irrelevant in the reduction procedure.

- Finally, we have also checked this method by applying it to a discussion of the gauge reduction of the conformal particle (in the Lagrangian formalism).

\section{A Linear reduction}

In this appendix we wish carry out a quick review of the reduction theory, giving at the same time a linear algebraic interpretation of this theory for the general case of linear forms of arbitrary order.

Let $E$ be a linear vector space, with $\operatorname{dim} E=n$, and a linear form $\alpha \in \Lambda^{k} E^{*}$, with $k \geq 2$. Let $S$ be a subspace of $E$. Then take

$$
S^{\perp_{1}}:=\{u \in E \mid i(u) i(v) \alpha=0, \forall v \in S\} \equiv N
$$

let $j: N \hookrightarrow E$ be the natural inclusion and $\alpha_{N}:=j^{*} \alpha$.

If $v \in N \cap S$, then $i(u) i(v) \alpha=0$, for every $u \in N$, and therefore $v \in$ ker $\alpha_{N}$; that is, $N \cap S \subset \operatorname{ker} \alpha_{N} \subset N$. Then we have the projections

$$
N \stackrel{\pi_{1}}{\longrightarrow} N / N \cap S \stackrel{\pi_{2}}{\longrightarrow} N / \operatorname{ker} \alpha_{N}=(N / N \cap S) /\left(\operatorname{ker} \alpha_{N} / N \cap S\right)
$$

and there exist $\alpha_{1} \in \Lambda^{k}(N / N \cap S)$ and $\alpha_{3} \in \Lambda^{k}\left(N / \operatorname{ker} \alpha_{N}\right)$ such that $\alpha_{N}=\pi_{1}^{*} \alpha_{1}$ and $\alpha_{N}=\pi_{3}^{*} \alpha_{3}$, where $\pi_{3}=\pi_{2} \circ \pi_{1}$.

Notice that ker $\alpha_{3}=\{0\}$, because the space $S$, projected by $\pi_{3}$, "has been removed". This is a "reduction" procedure in the sense that a subspace is removed from a vector space by a reduction of the dimension. Note that it is not useful to make the quotient $E / S$ and then the projection $E \rightarrow E / S$ because the form $\alpha$ does not project onto the quotient unless $S \subset \operatorname{ker} \alpha$. Then, $N=S^{\perp_{1}}$ is a subspace of $E$ which can be reduced in such a way that the form $\alpha$ goes down to the quotient.

As a particular situation, we can study the case $k=2$. Then we can prove that

$$
\operatorname{ker} \alpha_{N}=\operatorname{ker} \alpha+N \cap S
$$

In fact; let $\left\{e_{1}, \ldots, e_{k}\right\}$ be a basis of $S$. If $v \in \operatorname{ker} \alpha_{N}$, then $i(u) i(v) \alpha=0$, for every $u \in N$, and $N \subset$ ker $i(v) \alpha$. But $N=\cap_{j=1, \ldots, k}$ ker $i\left(e_{j}\right) \alpha$, then we have that $i(v) \alpha$ is a linear combination of $i\left(e_{1}\right) \alpha, \ldots, i\left(e_{k}\right) \alpha$ and, therefore, $v \in(\operatorname{ker} \alpha+S) \cap N$. But, since ker $\alpha \subset N$, the result follows.

If in addition, $\alpha$ is a symplectic linear form; that is, ker $\alpha=\{0\}$, then ker $\alpha_{N}=N \cap S$ and we have the unique projection

$$
N \stackrel{\pi}{\longrightarrow} N / N \cap S
$$

and a unique form $\hat{\alpha} \in \Lambda^{2}(N / N \cap S)$ with $\pi^{*} \hat{\alpha}=\alpha$, which is also a symplectic form. This is the result of the Marsden-Weinstein reduction procedure in the linear case.

\section{Acknowledgments}

We thank Dr. Xavier Gràcia-Sabaté (U.P.C.) for bringing the example of the conformal particle to our attention and for explaining to us some of its characteristics. We are greateful for the assistance 
of the referee, whose suggestions have enabled us to improve the final version of the work. We also thank Mr. Jeff Palmer for his assistance in preparing the English version of the manuscript.

We want also to thank the financial support of the CICYT TAP97-0969-C03-01.

\section{References}

[1] R. Abraham, J.E. Marsden, Foundations of Mechanics (2nd ed.), Addison-Wesley, Reading, 1978.

[2] R. Abraham, J.E. Marsden, T. Ratiu, Manifolds, Tensor Analysis, and Applications, Addison-Wesley, Reading, MA., 1983.

[3] C. Albert, "Le théorème de réduction de Marsden-Weinstein en géométrie cosymplectique et de contact", J. Geom. Phys. (1989) 627-649.

[4] J.M. Arms, R.H. Cushman, M.J. Gotay, "A Universal Reduction Procedure for Hamiltonian Group Actions", T. Ratiu Ed., M.S.R.I. Series 22, Springer-Verlag (1991) 33-52.

[5] M. Asorey, J.F. Cariñena, L.A. Ibort, "Generalized canonical transformations for timedependent systems", J. Math. Phys. 24 (1983) 2745.

[6] L. Bates, J. Sniatycki, "Non-holonomic Reduction", Rep. Math. Phys. 32 (1993) 99-115.

[7] M.J. Bergvelt, E.A. De Kerf, "Yang-Mills theories as constrained Hamiltonian systems", Physica A 139 (1986) 101-124.

[8] R.L. Bryant, S.S. Chern, R.B. Gardner, H.L. Goldschmidt, P.A. Griffiths, Exterior Differential Systems, Springer-Verlag, New York, 1991.

[9] F. Cantrijn, J.F. Cariñena, M. Crampin, L.A. Ibort, "Reduction of degenerate Lagrangian systems", J. Geom. Phys. 3(3) (1986) 353-400.

[10] F. Cantrijn, M. de León, J.C. Marrero, D. Martín de Diego, "Reduction of nonholonomic mechanical systems with symmetries", Rep. Math. Phys. 42(1-2) (1998) 25-45.

[11] A. Capri, M. Kobayashi, "A Mechanical Model with Constraints", J. Math. Phys. 23 (1982) 736-741.

[12] A. Capri, M. Kobayashi, "The first-rank tensor field coupled to an electromagnetic field", J. Phys. A: Math. Gen. 20 (1987) 6101-6112.

[13] J.F. Cariñena, "Theory of singular Lagrangians", Fortschr. Phys. 38 (1990) 641-679.

[14] J.F. Cariñena, J. Gomis, L.A. Ibort, N. Román-Roy, "Canonical transformation theory for presymplectic systems", J. Math. Phys. 26 (1985) 1961-1969.

[15] J.F. Cariñena, J. Gomis, L.A. Ibort, N. Román-Roy, "Applications of the canonical transformation theory for presymplectic systems", Nuovo Cim. B. 98 (1987) 172-196.

[16] J.F. Cariñena, L.A. Ibort, E.A. Lacomba, "Time scaling as an infinitesimal canonical transformation", Cel. Mec. 42 (1988) 201-213.

[17] W. Cox, "Lagrangian presymplectic constraint analysis of mechanical models of field theories coupled to external fields", J. Phys. A: Math. Gen. 25 (1992) 4443-4457.

[18] M. Crampin, G.E. Prince, G. Thompson, "A geometrical version of the Helmholtz conditions in time-dependent Lagrangian dynamics", J. Phys. A: Math. Gen. 17 (1984) 1437-1447.

[19] P.A.M. Dirac, "Generalized Hamiltonian dynamics", Canad. J. Math. 2 (1950) 129-148. 
[20] A. Echeverría-Enríquez, M.C. Muñoz-Lecanda, N. Román-Roy, "Geometrical setting of time-dependent regular systems. Alternative models", Rev. Math. Phys. 3(3) (1991) 301-330.

[21] A. Echeverría-Enríquez, M.C. Muñoz-Lecanda, N. Román-Roy, "Non-standard connections in classical mechanics", J. Phys. A: Math. Gen. 28 (1995) 5553-5567.

[22] M.J. Gotay, Presymplectic Manifolds, Geometric Constraint Theory and the DiracBergmann Theory of Constraints, Ph. D. Thesis, Univ Maryland, 1979

[23] M.J. Gotay, J.M. Nester, G. Hinds, "Presymplectic manifolds and the Dirac-Bergmann theory of constraints", J. Math. Phys. 27 (1978) 2388-2399.

[24] M.J. Gotay, "On coisotropic imbeddings of presymplectic manifolds", Proc. Amer. Math. Soc. 84 (1982) 111-114.

[25] X. Gràcia, J.M. Pons, "A generalized geometric framework for constrained systems", Diff. Geom. Appl. 2 (1992) 223-247.

[26] X. GrÀcia, J. RocA, "Covariant and noncovariant gauge transformations for the conformal particle", Mod. Phys. Let. 19 (1993) 1747-1761.

[27] H. Hamoni, A. Lichnerowicz, "Geometry of the dynamical systems with time-dependent constraints and time-dependent Hamiltonian: an approach towards quantization", J. Math. Phys. 25 (1984) 923-934.

[28] L.A. Ibort, J. Marín-Solano, "A geometric classification of Lagrangian functions and the reduction of the evolution space", J. Phys. A: Math. Gen. 25 (1992) 3353-3367.

[29] L.A. Ibort, J. Marín-Solano, "Coisotropic regularization of singular Lagrangians", J. Math. Phys. 36(10) (1995) 5522-5539.

[30] R. Kuwabara, "Time-dependent mechanical symmetries and extended Hamiltonian systems ", Rep. Math. Phys. 19 (1984) 27-38.

[31] M. De León, D. Martín de Diego, "Almost Product Structures and Poisson Reduction of presymplectic Systems", Extracta Math. 10(1) (1995) 37-45.

[32] M. DE León, M.H. Mello, P.R. Rodrigues, "Reduction of degenerate non-autonomous Lagrangian systems" Mathematical Aspects of Classical Field Theory, Contemp. Math. 132, 275-305, Eds. M.J. Gotay, J.E. Marsden, V. Moncrief, Seattle, Washington (USA), 1992.

[33] M. De León, M. Saralegi, "Cosymplectic reduction for singular momentum maps", J. Phys. A: Math. Gen. 22 (1993) 1-11.

[34] P. Libermann, C.M. Marle, Symplectic geometry and analytical dynamics, D. Reidel Publishing Company, Dordrecht, 1987.

[35] C.L. MARle, "Sous-variétés de rang constant d'une varieté symplectique", Astérisque 107108 (1983) 69-87.

[36] C.L. Marle, "Reduction of Constrained Mechanical Systems and Stability of Relative Equilibria", Comm. Math. Phys. 174 (1995) 295-318.

[37] G. Marmo, E.J. Saletan, A. Simoni, B. Vitale, Dynamical Systems, a Differential Geometric Approach to Symmetry and Reduction. J. Wiley, N.Y. 1985.

[38] R. Marnelius, "Manifestly conformally covariant description of spinning and charged particles", Phys. Rev. D 20 (1979) 2091.

[39] J.E. Marsden, T.S. Ratiu, "Reduction of Poisson manifolds", Lett. Math. Phys. 11 (1986) 161-170. 
A. ECHeverRía et al: Reduction of presymplectic manifolds with symmetry.

[40] J.E. Marsden, T.S. Ratiu, A. Weinstein, "Semi-direct Products and Reduction in Mechanics", Trans. Am. Math. Soc. 281 (1984) 147-177.

[41] J.E. Marsden, J. Scheurle, "The Reduced Euler-Lagrange Equations", Fields Inst. Comm. 1 (1993) 139-164.

[42] J.E. Marsden, A. Weinstein, "Reduction of symplectic manifolds with symmetry", Rep. Math. Phys. 5 (1974) 121-130.

[43] M.C. MuÑoz-LeCAndA, "Hamiltonian systems with constraints: a geometric approach". Int. J. Theor. Phys. 28 (11) (1989) 1405-1417.

[44] M.C. Muñoz-Lecanda, N. Román-Roy, "Lagrangian theory for presymplectic systems", Ann. Inst. H. Poincaré A 57(1) (1992) 27-45.

[45] M.C. Muñoz-Lecanda, N. Román-Roy, "Gauge systems: presymplectic and group action formulations", Int. J. Theor. Phys. 32(11) (1993) 2077-2085.

[46] T. Okubo, Differential Geometry, Monographs in Pure and Applied Mathematics 112, N.Y., 1987

[47] M.F. RAÑADA, "Extended tangent bundle formalism for time-dependent Lagrangian systems", J. Math. Phys. 32 (2) (1991) 500-505.

[48] W. Siegel, "Conformal invariance of extended spinning particle mechanics", Int. J. Mod. Phys. A 3 (1988) 2713.

[49] R. Sjamaar, E. Lerman, "Stratified symplectic spaces and reduction", Ann. Math. 134 (1991) 375-422.

[50] J.M. Souriau, Structure des systèmes dynamiques, Dunod, Paris, 1969.

[51] F.W. WARner, Foundations on differentiable manifolds and Lie groups, Scott, Foresman and Co., Glenview, 1971.

[52] A. Weinstein, Lectures on symplectic manifolds, C.B.M.S. Reg. Conf. Ser. Math., 29, 1979. 